\title{
Three clusters of the SMC from ACS/WFC HST archive data: NGC 265, K 29 and NGC 290 and their field population
}

\author{
E. Chiosi ${ }^{1}$ and A. Vallenari ${ }^{2}$
}

\author{
1 Astronomy Department, Padova University, Vicolo dell'Osservatorio 2, 35122 Padova, Italy \\ e-mail: emanuela.chiosi@oapd.inaf.it \\ 2 INAF, Padova Observatory, Vicolo dell'Osservatorio 5, 35122 Padova, Italy \\ e-mail: antonella.vallenari@oapd.inaf.it
}

Received 28 November 2006 / Accepted 28 January 2007

\begin{abstract}
Aims. We determine the age, metallicity and initial mass function of three clusters, namely NGC 265, K 29, NGC 290, located in the main body of the Small Magellanic Cloud. In addition, we derive the history of star formation in the companion fields.

Methods. We make use of ACS/WFC HST archive data. For the clusters, the age and metallicity are derived fitting the integrated luminosity function with single synthetic stellar population by means of the $\chi^{2}$ minimization. For the companion fields, the history of star formation is derived using the $\chi^{2}$ minimization together with the downhill-simplex method.

Results. For the clusters we find the following ages and metallicities: NGC 265 has $\log ($ Age $)=8.5 \pm 0.3 \mathrm{yr}$ and metallicity $0.004 \pm$ $0.003($ or $[\mathrm{Fe} / \mathrm{H}]=-0.62) ; \mathrm{K} 29$ has $\log (\mathrm{Age})=8.2 \pm 0.2 \mathrm{yr}$ and metallicity $Z=0.003 \pm 0.002($ or $[\mathrm{Fe} / \mathrm{H}]=-0.75) ; \mathrm{NGC} 290 \mathrm{has}$ $\log ($ Age $)=7.8 \pm 0.5 \mathrm{yr}$ and metallicity $0.003 \pm 0.002($ or $[\mathrm{Fe} / \mathrm{H}]=-0.75)$. The superior quality of the data allows the study of the initial mass function down to $M \sim 0.7 M_{\odot}$. The initial mass function turns out to be in agreement with the standard Kroupa model. The comparison of the NGC 265 luminosity function with the theoretical ones from stellar models both taking overshoot from the convective core into account and neglecting it, seems to suggest that a certain amount of convective overshoot is required. However, this conclusion is not a strong one because this cluster has a certain amount of mass segregation which makes it difficult to choose a suitable area for this comparison. The star formation rate of the field population presents periods of enhancements at 300-400 Myr, 3-4 Gyr and finally 6 Gyr. However it is relatively quiescent at ages older than 6 Gyr. This result suggests that at older ages, the tidal interaction between the Magellanic Clouds and the Milky Way was not able to trigger significant star formation events.
\end{abstract}

Key words. galaxies: Magellanic Clouds - galaxies: star clusters - galaxies: stellar content galaxies: luminosity function, mass function

\section{Introduction}

It is well known that the rich cluster system of the Magellanic Clouds (MCs) is quite different from that of our own Galaxy where a dichotomy is found between young sparse open clusters and old compact objects. The MCs present a wide distribution of rich cluster ages and constitute an ideal laboratory for testing the star and cluster formation process, either due to global effects, such as gravitational triggers because of tidal interactions, or to internal processes. In fact, the star formation (SF) process depends on the cooling and the heating of the interstellar gas which in turn, depend on the presence of metals (Lisenfeld \& Ferrara 1998; Wolfire et al. 1995). The Large and Small Magellanic Clouds (LMC and SMC, respectively) are particularly important as they allow us to study the SF in low metal content environments. A large population of clusters is found in the SMC. Hodge (1986), comparing the number of clusters found down to $B=22$ (inner regions) or $B=23$ (outer regions) in selected regions with the number of known clusters in SMC catalogs at that time, estimated a global population of 900 clusters, but more than 2000 clusters are expected if small-size, older clusters were detectable.

Concerning the cluster age distribution, several catalogs are available in literature. Pietrzynski \& Udalski (1999) using isochrone fitting, and Rafelski \& Zaritsky (2005) making use of integrated colors derive the age of a limited number of bright clusters, namely 93, and 200 respectively. Chiosi et al. (2006) derive the age of about 164 associations and 300 clusters using isochrone fitting on OGLE II data. However only a few of the clusters in those catalogs have ages derived from high quality CMD data. Ground based CMDs must avoid the cluster centers and suffer from the effects of the high crowding and serious contamination by field stars. The superior quality of Hubble Space Telescope (HST) data favors the study of the central regions of the clusters allowing precise determinations of their ages. Among the few age determinations of SMC clusters based on HST data, we quote Rich et al. (2000) who presented HST/WFPC2 data of 7 clusters.

In this paper, as a part of a project aimed to cast light on the process of field and cluster formation in the MCs, we present HST ACS/WFC archive data of three SMC clusters, namely NGC 265, K 29 and NGC 290 observed by Olszewski in 2004 (Proposal ID $=10126$ ) as part of the ACS/WFC program, for which no high quality CMDs have been obtained up to now. They are located close to the borders of HI shell 37A where star formation seems to have been very active in the recent past (Stanimirović et al. 2004; Chiosi et al. 2006). The coordinates of the clusters are given in Table 1. Preliminary age determinations on the basis of OGLE II data and isochrone fitting can be found in Chiosi et al. (2006) who, assuming the typical value for the metallicity $(Z=0.004)$ and the reddening appropriate to each 
Table 1. Equatorial coordinates of the SMC regions containing the three clusters.

\begin{tabular}{lcc}
\hline \hline Cluster & RA (J2000) & Dec (J2000) \\
\hline NGC 265 & $0: 47: 12$ & $-73: 28: 38$ \\
K 29 & $0: 51: 53$ & $-72: 57: 14$ \\
NGC 290 & $0: 51: 14$ & $-73: 09: 41$ \\
\hline
\end{tabular}

clusters, estimated $\log ($ Age $)=8.4,8.0,7.8 \mathrm{yr}$ for NGC 265, K 29, and NGC 290, respectively.

The aim of this paper is to derive better estimates of the age and metallicity of the three clusters, to check the slope of their initial mass functions (IMF) down to $M \sim 0.7 M_{\odot}$, and to derive the star formation history (SFH) of the companion field populations.

In Sect. 2 we present the data and describe the reduction procedure. In Sect. 3 we discuss the SMC distance modulus, the reddening and the metallicity. In Sect. 4 we describe the techniques used to derive age, metal content, and IMF. In Sect. 5 we derive the physical parameters of the clusters. In Sect. 6 we determine the SFH of the nearby fields. Finally, in Sect. 7 we draw the final remarks.

\section{Data presentation, reduction and calibration}

Reduction. We make use of HST archive data taken in 2004 using the Advanced Camera Survey (ACS/WFC) in the filters $F 555 \mathrm{~W}$ and $F 814 \mathrm{~W}$. Two exposure times per filter are available, namely $120 \mathrm{~s}$ and $440 \mathrm{~s}$. We make use of pre-reduced data that are already corrected for the geometric distortion of the camera. The data reduction is made with the DAOPHOT II/ALLSTAR packages by Stetson (1994). In brief, the CCD images are split in two regions, one containing the cluster and the other the field, the aperture photometry is calculated with an aperture of 3 pixels, the point spread function (PSF) is derived for each colour and each CCD, and the PSF photometry is performed. Data are finally corrected for the charge transfer efficiency (CTE) using the Riess \& Mack (2004) transformations. This correction goes from $0.01 \mathrm{mag}$ for the brightest magnitudes to 0.1 mag for the faintest magnitudes.

Calibration. The transformation from the instrumental magnitudes to the ACS/WFC-Vega system, taken from Bedin et al. (2005), is given by

$$
\begin{aligned}
m_{\text {filter }} \equiv & -2.5 \log _{10} \frac{I_{e^{-}}}{\text {exptime }}+Z_{\mathrm{p}}^{\text {filter }} \\
& -\Delta m_{\mathrm{PSF}-\mathrm{AP}(r)}^{\text {filter }}-\Delta m_{\mathrm{AP}(r)-\mathrm{AP}(\infty)}^{\text {filter }}
\end{aligned}
$$

where exptime is the exposure time, $Z_{\mathrm{p}}^{F 555}=25.718$ and $Z_{\mathrm{p}}^{F 814}=$ 25.492 (Bedin et al. 2005), and $\Delta m_{\mathrm{PSF}-\mathrm{AP}(r)}^{\text {filter }}$ and $\Delta m_{\mathrm{AP}(r)-\mathrm{AP}(\infty)}^{\text {filter }}$ are the two terms of the aperture correction. The first one compares the PSF magnitudes with the aperture magnitudes at a finite magnitude ( 3 pixels in our case) and the second one is the difference between aperture magnitude at finite size and magnitude at infinite aperture. The first term, which is tabulated in Table 2 separately for the two parts in which each CCD frame has been split, is statistically calculated for the brightest magnitudes as the mean difference. We assume as aperture corrections from $r=3$ pixel to infinity the value given by Sirianni et al. (2005): $\Delta m_{\mathrm{AP}(r)-\mathrm{AP}(\infty)}=0.243$ for the $F 555 \mathrm{~W}$ filter, $\Delta m_{\mathrm{AP}(r)-\mathrm{AP}(\infty)}=0.291$ for the $F 814 W$ filter.

ACS/WFC magnitudes are converted to the JohnsonCousins $V, I$ following Sirianni et al. (2005). The magnitudes are
Table 2. Aperture corrections for both filters $F 555 W$ and $F 814 W$. CCDs are split in 2 parts: the upper row (1) refers to the cluster whereas the lower row (2) to the field.

\begin{tabular}{llll}
\hline \hline Cluster & & $\Delta m_{\mathrm{PSF}-\mathrm{AP}(3 \mathrm{p} \text { ix })}^{F 555 \mathrm{~V}}$ & $\Delta m_{\mathrm{PSF}-\mathrm{AP}(3 \mathrm{pix})}^{F 814 W}$ \\
\hline NGC 265 & $(1)$ & $-0.14 \pm 0.02$ & $-0.18 \pm 0.04$ \\
& $(2)$ & $-0.15 \pm 0.02$ & $-0.21 \pm 0.03$ \\
K 29 & $(1)$ & $-0.14 \pm 0.02$ & $-0.19 \pm 0.02$ \\
& $(2)$ & $-0.15 \pm 0.02$ & $-0.19 \pm 0.03$ \\
NGC 290 & $-(1)$ & $-0.14 \pm 0.02$ & $-0.18 \pm 0.02$ \\
& $(2)$ & $-0.13 \pm 0.02$ & $-0.18 \pm 0.03$ \\
\hline
\end{tabular}

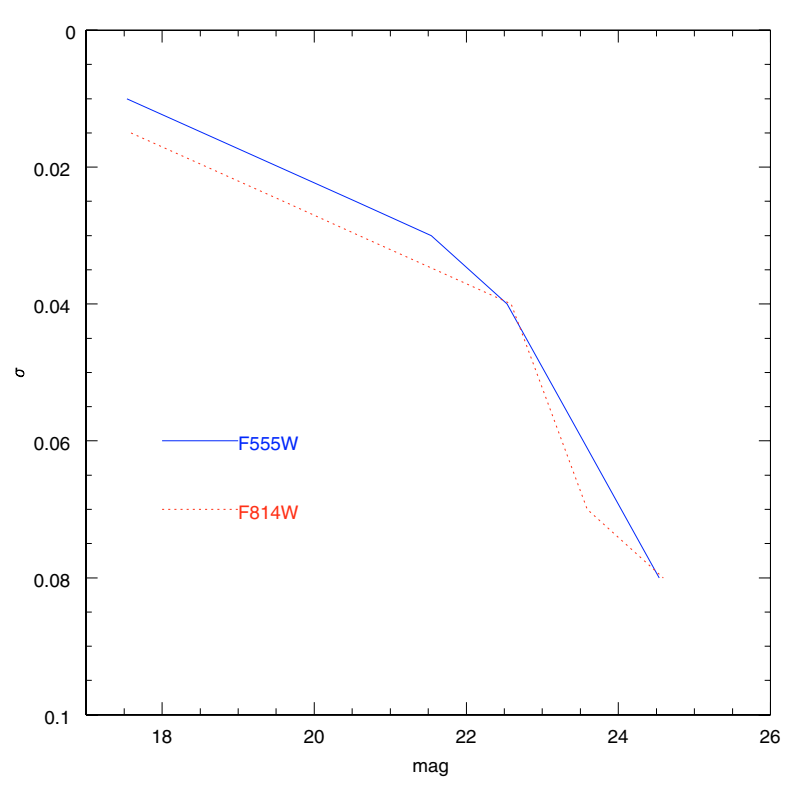

Fig. 1. Mean photometric errors $\sigma$ as function of the magnitude for the $F 555 W$ and $F 814 W$ pass-bands for the studied fields.

compared with $V, I$ ground-based data from OGLE II (Udalski et al. 1998) survey for NGC 265, K 29 and NGC 290. We make use of the stars brighter than $V=18$ and fainter than $V=17.3$ to avoid deviation from linearity due to saturation on HST data. The mean magnitude differences are $\Delta_{V}=V($ HST $)-$ $V($ OGLEII $)=0.02 \pm 0.04$ and $\Delta_{I}=I($ HST $)-I($ OGLEII $)=$ $0.01 \pm 0.04$. These small differences allow us to take the magnitudes of the saturated stars in the ACS photometry from the OGLE II catalog (see the sections below for more detail).

We estimate the photometric errors in the two pass-bands by means of the artificial stars experiments as the difference between the assigned and recovered magnitude for any simulated star. The results are shown in Fig. 1 as a mean of all the studied fields. This is justified by the fact that differences from field to field are not relevant.

The observational CMDs of the three clusters are presented in the top left panels of the Figs. 2-4. They will be examined in detail below. They refer to a circular area having $22-25^{\prime \prime}$ of radius (depending on the cluster) inside which the vast majority of the cluster stars falls as shown by the surface brightness profiles in the top right panels of the Figs. 2-4 (see the discussion below).

Incompleteness. Correction for incompleteness is derived as usual by means of artificial star experiments where artificial stars of known magnitude are added to the original image and data are re-reduced. The ratio between the number of recovered stars and the original number of added stars gives the completeness correction $\Lambda$. The results are shown in the bottom right panels 

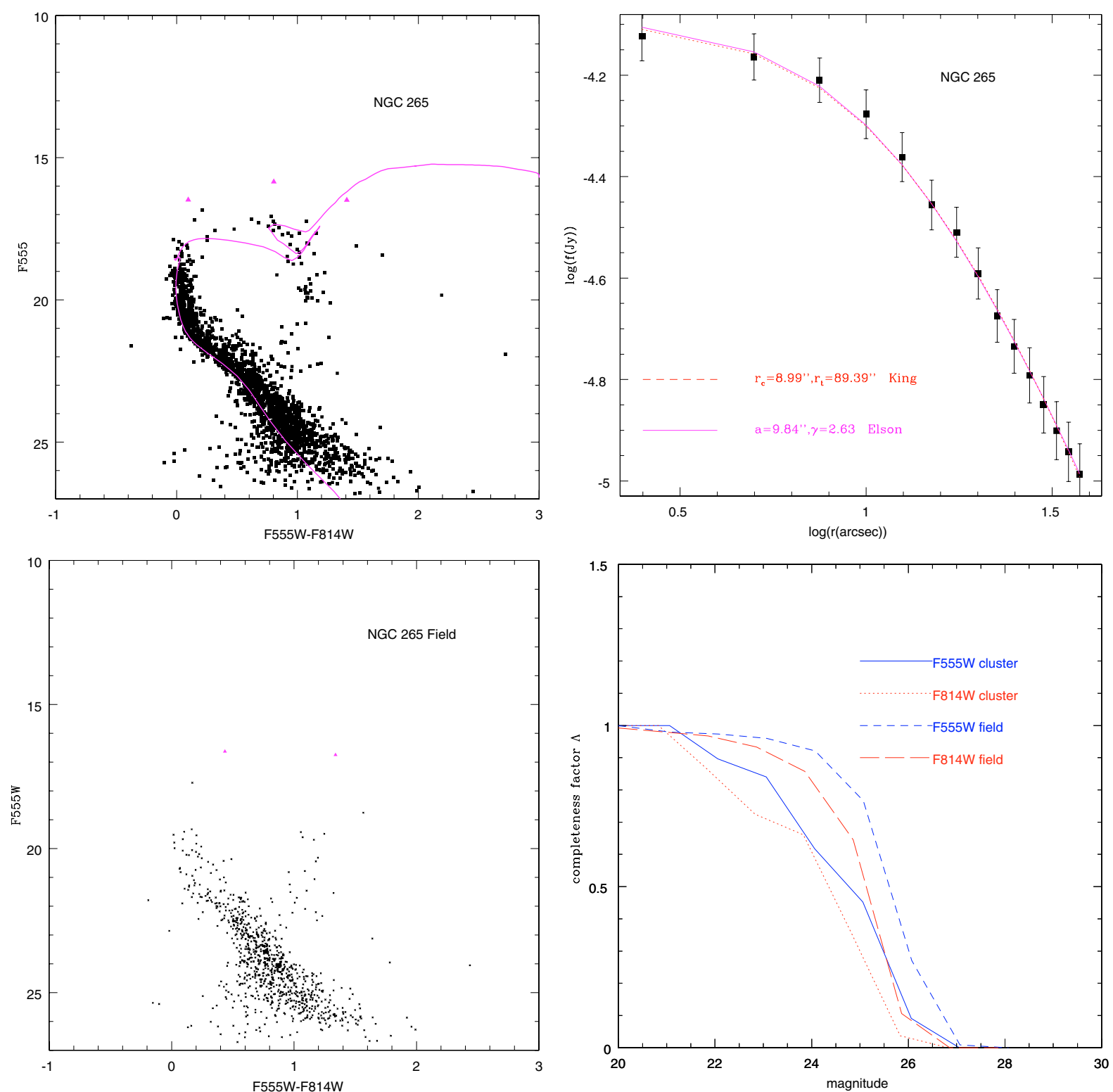

Fig. 2. Summary panels for NGC 265. In the top left panel is the CM diagram (inner $\left.22^{\prime \prime}\right)$ with a superposed isochrone of $\log (\operatorname{age})=8.5, Z=0.004$ and $E(F 555 W-F 814 W)=0.08$. The brightest stars settle at $F 555 W=16$. In the top right panel the surface brightness profile (in arbitrary units) is fitted by one of the King functions (dashed line) and by Elson profile (continuous line). From this distribution we can determine core and tidal radius. Vast majority of the stars sets within a radius of $22^{\prime \prime}$ corresponding to $\log (r)=1.3$. In the bottom left panel the CMD of field stars is shown. The field population is taken at about $1.6^{\prime}$ from the cluster center and the area is comparable to the area of the cluster (see text for details). The bottom right panel shows the completeness factors for the cluster and field regions as indicated.

of the Figs. 2-4 which present $\Lambda$ as function of the magnitude for the inner radius of the clusters. and for the external regions. More details can be found in the sections below.

\section{Distance modulus, reddening and metallicity}

The distance modulus $(m-M)_{0}=18.9$ is assumed for the SMC, in agreement with recent determinations by Storm et al. (2004), Weldrake et al. (2004), Caputo et al. (1999) and Sandage et al. (1999).

No assumptions are made for the reddening and metallicity of each cluster. They are derived together with the age from the analysis of the CMD by means of the $\chi^{2}$ minimization technique to be discussed below.
In the study of the field population, assumptions have to be made for the SMC depth along the line of sight, interstellar extinction and age-metallicity relation. We proceed as follows:

1. We adopt the SMC depth along the line of sight of $4 \mathrm{kpc}$, which implies a difference in the distance modulus of about $0.14 \mathrm{mag}$ (Welch et al. 1987; Zaritsky et al. 2002).

2. The extinction map across the SMC derived by Zaritsky et al. (2002) shows that the interstellar reddening is spatially varying, i.e. increasing along the SMC ridge from North-East to South-West. A mean reddening $E(B-V)=0.08 \mathrm{mag}$ is derived by Rachford et al. (2002) and Hunter et al. (2003). However, we prefer to use for the field population the same extinction we have obtained for the study of the CMD of the companion cluster using the minimization procedure. 

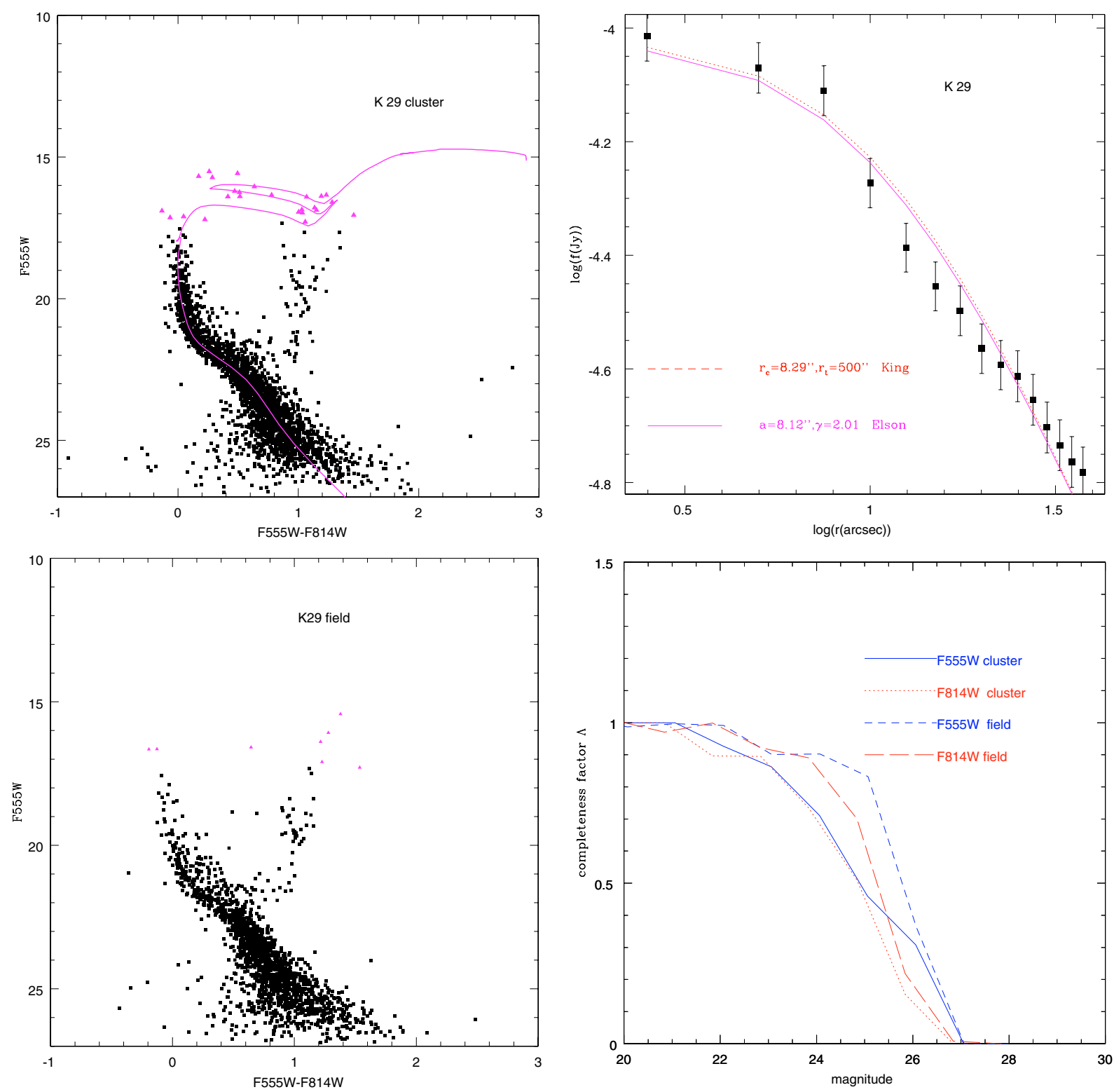

Fig. 3. Summary panels for K 29. In the top left panel is the CM diagram (inner $22^{\prime \prime}$ ) with a superposed isochrone of $\log ($ age $)=8.2, Z=0.003$ and $E(F 555 W-F 814 W)=0.14$. The OGLE bright stars are indicated by the triangles. The brightest stars settle at $F 555 W=15$. In top right is the brightness profile (in arbitrary units) fitted with one of the King (dashed line) and with the Elson functions (solid line). From this distribution we can determine core and tidal radius. Vast majority of the stars sets within a radius of $22^{\prime \prime}($ or $\log (r)=1.3)$. In the bottom left panel the CMD of field stars is shown. The field population is taken at about $1.6^{\prime}$ from the cluster center and the area is comparable to the area of the cluster (22"). The bottom right panel shows the completeness factors for the cluster and field regions as indicated.

3. Our knowledge of the age-metallicity relation in the SMC is mainly based on clusters. The interpretation of the existing SMC age-metallicity relation widely varies from author to author. It goes from continuous enrichment from the oldest to the youngest objects as found by Da Costa \& Hatzidimitriou (1998) and by Dolphin (2000b) to a burst-mode of star formation and enrichment as derived by Olszewski et al. (1996), Pagel \& Tautvaišienè (1999), Piatti et al. (2001), Harris $\&$ Zaritsky (2004). To study the star formation history in the fields, we assume the enrichment history by Pagel \& Tautvaišienè (1999).

\section{Method to derive ages, metallicities, and IMFs}

Age, metal content, and IMF are estimated by comparing theoretical luminosity functions (LF) that depend on the three parameters under examination to the observational one and by plotting the corresponding isochrones on the CMD to check the mutual consistency of the results. The determination is first made by visual inspection of the CMD and it is refined with the aid of the $\chi^{2}$ minimization. Theoretical luminosity functions and isochrones are taken from the library of Girardi et al. (2002).

Corrections for completeness and field subtraction are the preliminary step to be undertaken to derive good experimental luminosity functions. The analysis articulates as follows:

1. Equal areas are considered for each cluster and companion field.

2. The luminosity interval spanned by the cluster and field stars in each pass-band is divided in number of intervals of 0.5 mag width. In each interval, and separately for the cluster and field, we count the stars and apply the correction for 

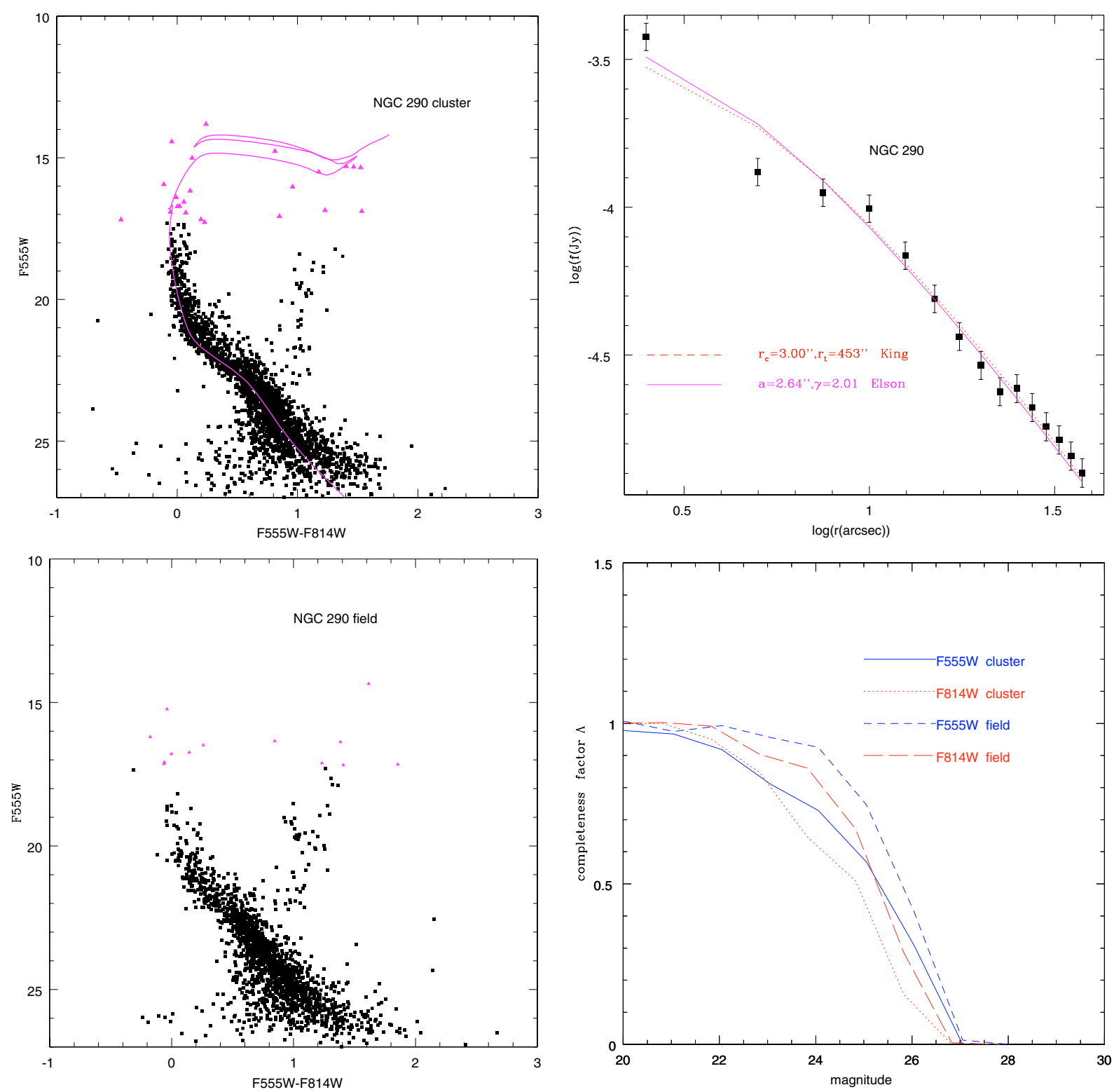

Fig. 4. Summary panels for NGC 290. In the top left panel is the CM diagram (inner radius of $25^{\prime \prime}$ ) with a superposed isochrone of log (age) $=7.8$, $Z=0.003, E(F 555 W-F 814 W)=0.15$. The OGLE bright stars are indicated by the triangles. The brightest stars settle at $F 555 W=14$. In top right is surface brightness profile (in arbitrary units) fitted with the King profile (dashed line) and with the Elson function (solid line). From this distribution we can determine the core and tidal radius. Vast majority of the stars sets within a radius of $25^{\prime \prime}$. In the bottom left panel the CMD of field stars is shown (see text for details). The field population is taken at about 1.6' from the cluster center and the area is comparable to the area of the cluster (25"). The bottom right panel shows the completeness factors for the cluster and field regions as indicated.

incompleteness (the cluster and field completeness factors are used as appropriate) and finally we calculate the ratio

$N_{\text {true }}=\frac{1}{\Lambda} \times N_{\text {obs }}$

where $\Lambda$ is the completeness factor.

3. In each magnitude interval the field stars are statistically subtracted from the cluster stars.

4. The above procedure is applied to derive both the LF and the CMD of the cluster. The corrected LFs for each cluster are presented in Figs. 7-9 together with their theoretical counterparts.

5. From the Girardi et al. (2002) isochrone library we select one of suitable age and metallicity that best matches the CMD paying particular care to the location of the main sequence, and to the luminosity of the core He-burning stars. Once the above estimates of age and metal content are obtained we refine them by means of the $\chi^{2}$ minimization of the LF. We start from the previous estimates, use the Padova simulator of synthetic CMDs, that can take the photometric errors on magnitudes into account, and apply the Monte Carlo technique to generate many simulations of the LF and companion CMD at varying age, metallicity, reddening, and slope of the IMF. Finally the $\chi^{2}$ minimization technique is applied to pin down the best combination of the parameters.

The IMF is derived in the mass range from the turnoff mass to the limit set by the completeness of the photometry, i.e. at about $0.7 M_{\odot}$. We assume an IMF of the form:

$\mathrm{d} N \propto M^{-\alpha} \mathrm{d} M$ 
where the slope $\alpha$ is a free parameter and is derived in two mass ranges, namely $0.7 M_{\odot}<M<1 M_{\odot}$ and $1 M_{\odot}<M<4 M_{\odot}$. In the adopted notation, the Salpeter (1955) slope is $\alpha=2.35$.

Binary stars. A large fraction of stars in cluster and field CMDs are unresolved binaries, either physical binaries or chance superposition of stars along the line of sight. Their presence blurs the main sequence, the giant branch, the clump of red stars, and the loop, thus making it more difficult to derive precise estimates of age, metallicity and IMF slope. Particularly cumbersome is the use of the turn-off or termination magnitude of the main sequence to infer the age by fitting isochrones and luminosity functions when binaries are present. The effect of unresolved binary stars on the CMD and luminosity functions of stellar clusters has been investigated in many studies among which we recall Chiosi et al. (1989a), Vallenari et al. (1991), and Barmina et al. (2002). As long known, the presence of unresolved binaries would first brighten the turn-off and termination magnitude and broaden the main sequence. Second if the photometry is particularly good they would even split the main sequence into two parallel loci, the one of unresolved binaries being systematically cooler and brighter (about 0.7 mag for binary stars of equal mass) than that of single stars, thus facilitating the task of assigning the true age and metallicity. Our present data do not allow this. Therefore we are left with the brightening of the turn-off and termination magnitude which would mimic a younger age and also yield a different slope for the IMF in the upper mass range. For the three clusters under examination we do not have information on their percentage of binary stars nor on the distribution of the mass ratio and separation of these latter. Despite this, we apply the synthetic CMD technique to include the effect of unresolved binaries. We assume that binary stars obey the same IMF of single stars and the mass ratio falls in the range 0.7 to 1 . Systems with mass ratios different from these cannot be excluded. However they would be hardly distinguishable from single objects. Finally, we suppose that the percentage of unresolved binaries is the same as indicated by open clusters of the Milky Way that amounts to about 30-50\% of the cluster population (Mermilliod \& Mayor 1989; Carraro et al. 1994), and other well studied clusters of the LMC such as NGC 1818 (Elson et al. 1998) and NGC 1866 (Barmina et al. 2002). Here we adopt the percentage of $30 \%$.

\section{Physical parameters of the clusters}

In this section, we first discuss the contamination by the field population, then we derive the brightness profile, and finally we determine age, metallicity, and IMF slope of each cluster.

\subsection{Field population contamination}

The sampling of the field population is a critical issue for surface profile determination and for the determination of the luminosity function. The field contamination of the three clusters is marginally different, (see Fig. 5) indicating an inhomogeneous distribution. For this reason and because of the relatively small size of the field of view of the ACS, we are forced to sample the field population close to the cluster center, i.e. within about $1.6^{\prime}$ from the cluster centers. To be sure that the cluster population does not significantly affect the field sample, we compare the LFs of the field population taken in this way with the field population taken from OGLE II data at a distance of about $1 \mathrm{deg}$. from the cluster centers down to a magnitude of $V=21$, where the completeness of the OGLE II data becomes critical. Figure 5 shows that, once that the LFs are normalized to an equal area,
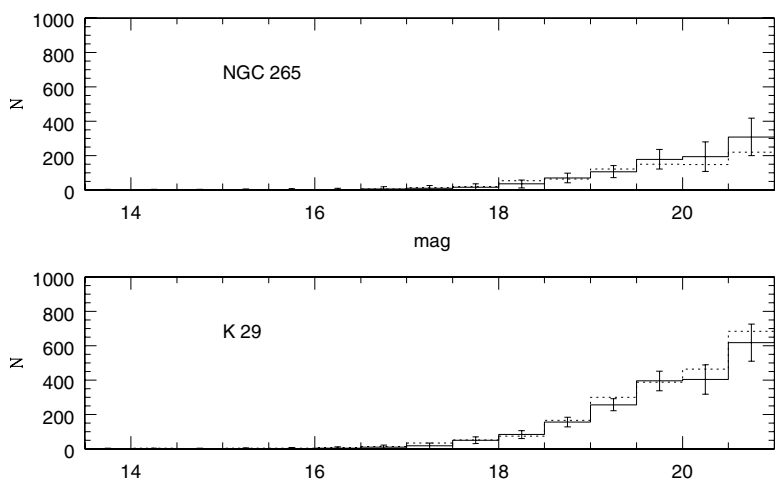

mag

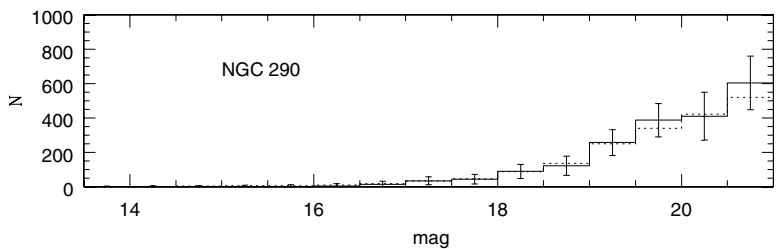

Fig. 5. The luminosity functions of HST (solid line) data and OGLE II (dashed line) data for the field area are compared. OGLE II field areas are taken at a distance of $1 \mathrm{deg}$ from the clusters. The number of objects is normalized to an area of $22^{\prime \prime}$.

close-by samples of field population are statistically comparable to the more distant ones. This suggests that the contamination by cluster stars inside the area selected to represent the field is probably not very relevant.

\subsection{Surface brightness profiles}

In this section, we derive the surface brightness profile of each cluster. The profiles are then compared with the theoretical ones of King (1962) and Elson et al. (1987) to determine the parameters of the clusters. First, pixel coordinates of each star are converted into the absolute coordinates $\alpha$ and $\delta$ using the task IRAF STSDAS "xy2rd". Then, the center of a cluster is determined calculating the luminosity weighted centroid at increasing radii. The photometric data of the stars that are saturated in the ACS/WFC observations are taken from OGLE II data, converted to the $F 555 \mathrm{~W}$ pass-band, and finally used to construct the surface brightness profile. We are confident that this does not introduce errors in the derived profiles as we have already checked the consistency between the OGLE II photometry in the $V$ magnitude and the ACS/WFC HST $V$ calibration.

Many SMC clusters have elliptical isophotes. This is not the case of the objects studied in this paper, which have only moderate ellipticity, $1-b / a \sim 0.13-0.3$ (Hill \& Zaritsky 2006). Thanks to this, we can use circular apertures to calculate the profile. Because of the high resolution of our images, the surface brightness given as $\log (f)$ inside the $i$-th circle having radius $b_{i}$ is obtained simply by adding up the flux of the stars falling inside the circle itself:

$\log (f)=1 /\left(\pi b_{i}^{2}\right) \times \sum_{j} \Lambda_{j} F_{j}$

where $\Lambda_{j}$ is the completeness factor inside the ring and $F_{j}$ the stellar flux. Crowding can cause a significant number of stars to be missed by ALLSTAR. Their flux can affect the surface brightness profile and must be accounted for. This is the reason why the completeness correction is applied to the flux determination. We restrict the determination of the surface profile to 
Table 3. Core and tidal radius as determined by the fit with the King (1962) and Elson et al. (1987) functions.

\begin{tabular}{l|lcc|cccc}
\hline \hline Cluster & \multicolumn{3}{|c|}{ King (1962) } & \multicolumn{4}{c}{ Elson et al. (1987) } \\
\hline & $r_{\mathrm{c}}$ & $\log \left(r_{\mathrm{t}} / r_{\mathrm{c}}\right)$ & $\chi^{2}$ & $r_{\mathrm{c}}$ & $a$ & $\gamma$ & $\chi^{2}$ \\
& $(\mathrm{pc})$ & & & $(\mathrm{pc})$ & $(\mathrm{pc})$ & & \\
\hline NGC 265 & 2.61 & 0.75 & 1.06 & 1.98 & 2.85 & 2.63 & 1.06 \\
K 29 & 2.40 & 2.50 & 1.27 & 2.35 & 2.35 & 2.01 & 1.16 \\
NGC 290 & 0.87 & 1.50 & 1.55 & 0.76 & 0.76 & 2.01 & 1.53 \\
\hline
\end{tabular}

the area completely falling inside the small field of view of the ACS/WFC camera. In all the cases, the profiles have maximum radii of $\log (r) \sim 1.5 \operatorname{arcsec}$.

The sky background subtraction is a critical step in the determination of the surface profile especially in the outer regions of a cluster. The field population is sampled as discussed in the previous section, the contamination is calculated as the mean flux in a field area of about $22^{\prime \prime}$, and is finally subtracted.

Two families of models are fitted on the brightness profiles. The first one is the tidally truncated functions of King (1962):

$f(x)=\pi r_{\mathrm{c}}^{2} k\left[\ln (1+x)-4 \frac{(1+x)^{1 / 2}-1}{\left(1+x_{\mathrm{t}}\right)^{1 / 2}}+\frac{x}{1+x_{\mathrm{t}}}\right]$

with $x=\left(r / r_{\mathrm{c}}\right)^{2}$ and $x_{\mathrm{t}}=\left(r_{\mathrm{t}} / r_{\mathrm{c}}\right)^{2}$ where $k$ is a constant and $r_{\mathrm{t}}$ and $r_{\mathrm{c}}$ are the searched parameters, specifically $r_{\mathrm{t}}$ is the tidal radius and $r_{\mathrm{c}}$ the core radius.

The second one is the Elson et al. (1987) family of functions:

$f(r)=\frac{2 \pi \mu_{0} a^{2}}{\gamma-2}\left[1-\left(1+\frac{r^{2}}{a^{2}}\right)^{1-\gamma / 2}\right]$

where $a$ is a scale length and $\gamma$ the power law index. These models are essentially the same as the King (1962) models, but they do not assume tidal truncation. The parameter $a$ is related to the King (1962) core radius $r_{\mathrm{c}}$ by:

$r_{\mathrm{c}}=a\left(2^{2 / \gamma}-1\right)^{1 / 2}$.

The profile fit is made using the $\chi^{2}$ minimization. The uncertainties on the profiles are calculated as Poisson errors.

\subsubsection{Results and comparison with previous work}

Figures 2-4 present the surface brightness profiles of each cluster and Table 3 gives the derived parameters. The core radii obtained using Elson et al. (1987) and King (1962) profiles are substantially in agreement, as expected because the main differences between the models arise at larger radii. The tidal radius is quite uncertain, first because it strongly depends on the sky subtraction and second, because our photometry does not extend to large enough radii. Table 4 compares our results on cluster parameters with those by Hill \& Zaritsky (2006). In comparison with our results, their values of $r_{\mathrm{c}}$ are in agreement for NGC 265, but they tend to be overestimated for the other two clusters, especially concerning the fit with Elson et al. (1987) profiles. The values of $\gamma$ by Hill \& Zaritsky (2006) are overestimated for all the clusters.

The question whether MC clusters are tidally limited or not is a long lasting one. The King (1962) models are expected to describe bound systems that have isothermal and isotropic stellar distribution functions and are limited by a strong tidal field. The
Table 4. Structural parameters of the clusters as determined by Hill \& Zaritsky (2006).

\begin{tabular}{l|llll}
\hline \hline \multicolumn{1}{c}{ Cluster } & \multicolumn{4}{c}{ Structural Parameters } \\
& \multicolumn{2}{c}{ King $(1962)$} & \multicolumn{2}{c}{ Elson et al. (1987) } \\
\hline & $r_{\mathrm{c}}$ & $r_{90}$ & $r_{\mathrm{c}}$ & $\gamma$ \\
& $(\mathrm{pc})$ & $(\mathrm{pc})$ & $(\mathrm{pc})$ & \\
\hline NGC 265 & 2.75 & 11.37 & 3.51 & 3.20 \\
K 29 & 1.22 & 14.58 & 5.63 & 6.00 \\
NGC 290 & 1.22 & 14.78 & 1.83 & 3.25 \\
\hline
\end{tabular}

Elson et al. (1987) models are empirically derived in order to reproduce the profiles of young MC clusters. The lack of tidal cutoff was originally explained with the dynamical youth of those clusters having extra-tidal stars. In this view, it is possible for a cluster to evolve or not from an Elson et al. (1987) profile to a King (1962) one, depending on the environment and on the cluster's characteristics. However, on an observational ground, no clear correlation is found between the age of the clusters and the presence of tidal cut-off. Hill \& Zaritsky (2006) find that the SMC clusters are fitted in a satisfactory way by both models, although the King (1962) profiles provide a slightly superior fit in $\sim 90 \%$ of the cases. McLaughlin \& van der Marel (2005) compare the King (1962) models, the Elson et al. (1987) profiles, and the modified isothermal spheres based on the Wilson (1975) models. These latter are spatially more extended than the King (1962) functions, but still include a finite, tidal cut-off in the density. The authors come to the conclusion that the un-truncated power-law distributions, Wilson (1975) models, and King (1962) profiles yield comparable good fits of MC clusters. McLaughlin \& van der Marel (2005) conclude that extended star envelopes around MC clusters may not be transient features due to the age, but more probably halos of self-gravitating objects. For the LMC clusters, Elson et al. (1987) find a clear trend that the spread in core radius is an increasing function of the cluster age. The observed trend probably represents a real structural evolution of the clusters: all clusters form with a relatively small radius and subsequently some of them experience a core expansion, while others do not. This result is confirmed by other authors (we quote as the most recent Mackey \& Gilmore 2003). To check whether the trend found for the LMC is a property of SMC clusters as well, we combine the cluster age determinations derived from isochrone fitting by Chiosi et al. (2006) with the values of the King (1962) core $r_{\mathrm{c}}$ obtained by Hill \& Zaritsky (2006). Figure 6 shows that a large spread in core size is present. From a theoretical point of view, while a core expansion during the evolution is expected as a consequence of the significant and rapid mass loss by stellar winds, it is less clear why a large scatter is present, corresponding to a more rapid expansion for some objects. Some mechanisms are proposed in literature which might influence the core evolution, such as variations in the stellar IMF, the effect of the surrounding tidal field or some external processes (i.e. clusters are not evolving in isolation), the presence of a varying population of primordial binaries. However, at present no satisfactory solution is found. A wider discussion of this problem is beyond the aims of this paper. It can be found in Elson (1991), Mackey \& Gilmore (2003), Wilkinson et al. (2003). It cannot be excluded that such a large scatter is due to observational uncertainties on the derived core radius (and ages) of the clusters. Better quality data are needed before reaching a firm conclusion. 


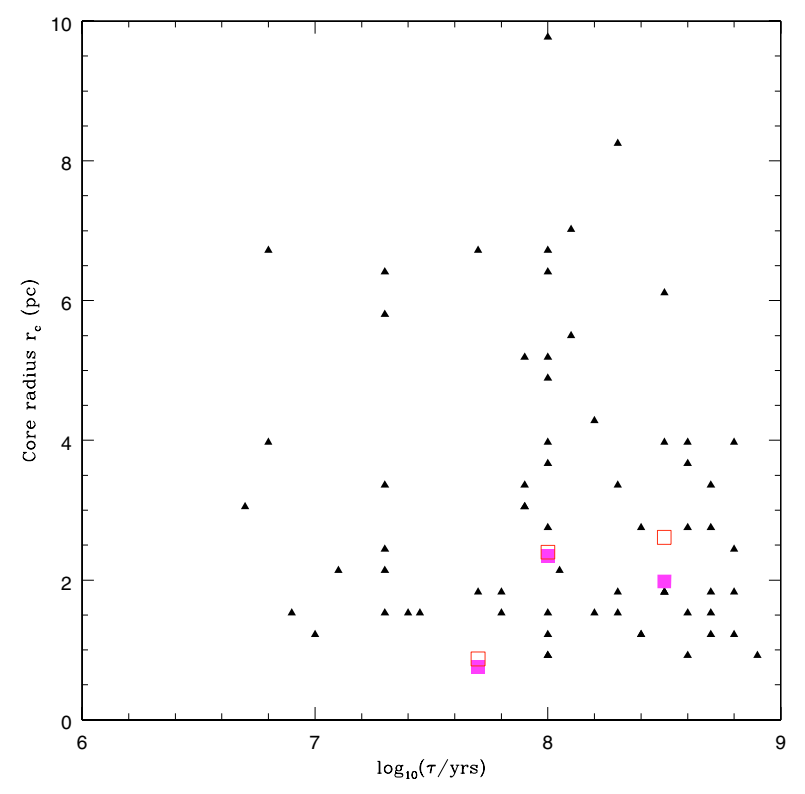

Fig. 6. The core radius versus age relationship for SMC clusters from the sample derived as described in the text. The three clusters studied in this work are plotted as filled squares for the Elson et al. (1987) models and empty squares for the King (1962) models.

Table 5. The best fit parameters, Age, $Z, E(F 555 W-F 814 W)$ for all the clusters.

\begin{tabular}{lccc}
\hline \hline Cluster & $\log ($ Age $)(\mathrm{yr})$ & $Z$ & $E(F 555 W-F 814 W)$ \\
\hline NGC 265 & $8.5 \pm 0.3$ & $0.004 \pm 0.003$ & $0.08 \pm 0.06$ \\
K 29 & $8.2 \pm 0.2$ & $0.003 \pm 0.002$ & $0.14 \pm 0.08$ \\
NGC 290 & $7.8 \pm 0.5$ & $0.003 \pm 0.002$ & $0.15 \pm 0.05$ \\
\hline Binaries \\
NGC 265 & $8.7 \pm 0.2$ & $0.004 \pm 0.003$ & $0.08 \pm 0.06$ \\
K 29 & $8.3 \pm 0.2$ & $0.003 \pm 0.002$ & $0.14 \pm 0.08$ \\
NGC 290 & $8.0 \pm 0.3$ & $0.003 \pm 0.002$ & $0.15 \pm 0.05$ \\
\hline
\end{tabular}

\subsection{Age, metallicity, IMF determinations}

In this section we derive the age, metal content, and IMF for each cluster. Table 5 presents the results for all the clusters. Those results are commented in the following Sections. Limited to the case of NCG 265, we also compare the CMD and LF with the predictions from models with and without overshoot from the convective core to further contribute to the long lasting debate on whether convective overshoot is an important phenomenon in stellar structure and evolution.

\subsubsection{NGC 265}

The cluster population is sampled in a circular region of $22^{\prime \prime}$ radius centered on the cluster. This radius is larger than the core radius of $\sim 18^{\prime \prime}$, thus ensuring that the cluster population is well represented. We sample the field population at 1.6' apart from the cluster center (see previous sections for a wider discussion of the field population). To subtract the field stars from the cluster LF, we consider a field area of $22^{\prime \prime}$. The field population is statistically subtracted from the LF and the CMD. Inside the radius of $22^{\prime \prime}$ about 1000 cluster stars are detected down to $F 555 \mathrm{~W}=25$ where the incompleteness reaches the $50 \%$ level. Starting from an initial guess obtained from visual inspection of the CMD, and using the CMD and LF simulator, by means of
Table 6. The best fit IMF coefficient in the range of low masses $\alpha_{1}$ $\left(0.7 M_{\odot}<M<1 M_{\odot}\right)$ and in the range of high masses $\alpha_{2}\left(M>1 M_{\odot}\right)$ is given for the three different clusters. The $68 \%$ confidence interval is also given $\left(\sigma_{1}\right.$ and $\left.\sigma_{2}\right)$.

\begin{tabular}{lcccc}
\hline \hline Cluster & $\alpha_{1}$ & $\sigma_{1}$ & $\alpha_{2}$ & $\sigma_{2}$ \\
\hline \multicolumn{5}{c}{ No binaries } \\
NGC 265 & 2.4 & 0.4 & 2.5 & 0.5 \\
K 29 & 1.8 & 0.2 & 2.7 & 0.3 \\
NGC 290 & 2.2 & 0.2 & 2.7 & 0.4 \\
\hline \multicolumn{5}{c}{ Binaries } \\
NGC 265 & 2.2 & 0.4 & 2.7 & 0.5 \\
K 29 & 2.2 & 0.2 & 2.7 & 0.3 \\
NGC 290 & 2.2 & 0.2 & 2.7 & 0.4 \\
\hline
\end{tabular}

the $\chi^{2}$ minimization we derive: $\log ($ Age $)=8.5 \pm 0.3 \mathrm{yr}$, interstellar reddening $E(F 555 W-F 814 W)=0.08 \pm 0.06$, corresponding to $A_{F 555 W}=0.21$ (Bedin et al. 2005), and finally metallicity $Z=0.004 \pm 0.003$. All the errors on the determinations of the parameters are given at the $68 \%$ confidence level. The top left panel of Fig. 2 presents the CMD where the isochrone for the age and metallicity we have determined is superposed. The isochrone gives a good fit of the observational CMD. Different combinations of extinction, metallicity and age (inside the quoted errors) give reasonable fits of the CMD. For instance, when $\log ($ Age $)=8.4 \mathrm{yr}, E(F 555 W-F 814 W)=0.1$, and $Z=0.007$, the main sequence color, the turnoff magnitude, and the luminosity of the loop of the core He-burning stars are well reproduced, while the theoretical loop is too red in comparison with the data. When $\log ($ Age $)=8.6 \mathrm{yr}, E(F 555 W-F 814 W)=0.15$, and $Z=0.001$, then the turnoff and the loop magnitudes and colors are well reproduced, but the colour of the unevolved part of the main sequence, two magnitudes about below the turnoff is poorly fitted. On the other hand, it is clear that these features of the CMD depend on the details of the stellar models (treatment of convection, mixing length, bolometric corrections, adopted chemical mixture...). For this reason, at some extent, the derived set of best fitting parameters is depending on the adopted stellar models. The turn-off mass is about $4 M_{\odot}$. The IMF slope in the mass range $M>1 M_{\odot}$ turns out to be $\alpha_{2}=2.5 \pm 0.5$, while in the mass range $\left(0.7<M<1 M_{\odot}\right)$ is $\alpha_{1}=2.4 \pm 0.4$, substantially in agreement with the Kroupa (2000) IMF. We recall that the Kroupa (2000) IMF has $\alpha_{1}=2.2$ for $0.5 M_{\odot}<M<1 M_{\odot}$, $\alpha_{2}=2.7$ for $M>1 M_{\odot}$.

When binary stars are included a slightly older age is found. We add a percentage of binaries amounting to $30 \%$ of the stars. Instead of an age of $\log (\operatorname{Age}(\mathrm{yr}))=8.5$ we find the best fit at an age of $\log (\operatorname{Age}(\mathrm{yr}))=8.7 \pm 0.2$. As a consequence, the determination of the exponents of the IMF changes. For all the studied clusters, the summary of the IMF determination is presented in Table 6. The quality of the fit is shown in Fig. 7 where the observational LF is compared with the best fit simulation.

\subsubsection{K 29}

We sample the cluster population inside a radius of $22^{\prime \prime}$ where about 700 stars are detected down to $F 555 W=25 \mathrm{mag}$. As for NGC 265, this radius is larger than the core radius. The field population of K 29 is located outside the core radius.

Figure 3 (top left panel) presents the CMD of the cluster (inside the $22^{\prime \prime}$ radius), where bright stars that are saturated in the ACS/WFC photometry are taken from the OGLE II data, analogously to the procedure adopted in the determination of the brightness. The age derived by $\chi^{2}$ minimization 


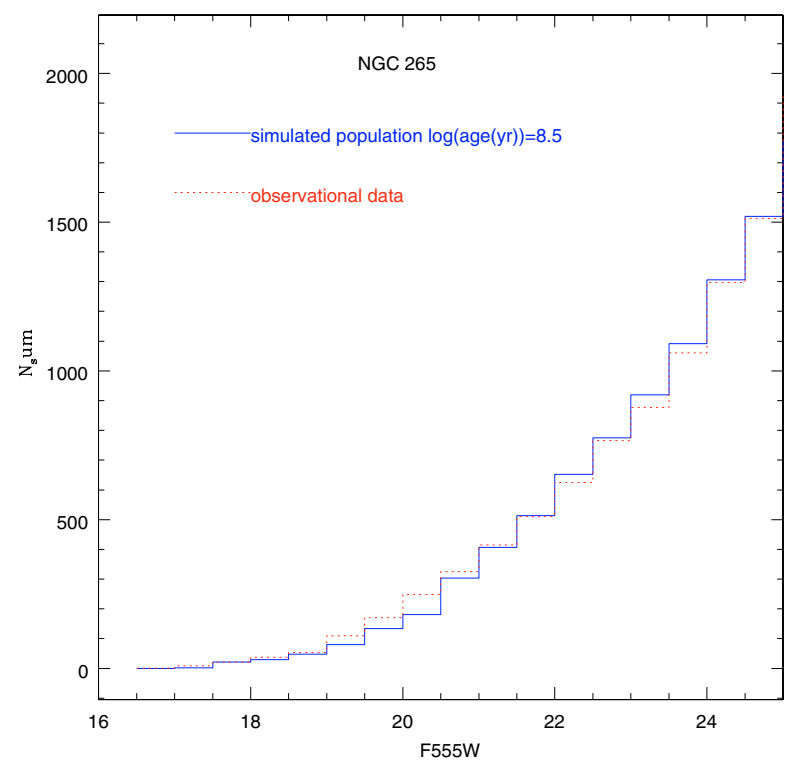

Fig. 7. $\mathrm{LF}$ of the cluster NGC 265 . The best fit is done with a population of $\log ($ Age $)=8.5 \mathrm{yr}$.

is $\log ($ Age $)=8.2 \pm 0.2 \mathrm{yr}$, the metallicity $Z=0.003 \pm 0.002$, and the reddening $E(F 555 W-F 814 W)=0.14 \pm 0.08$. The turn-off mass is about $4.5 M_{\odot}$. The slope of the IMF in the mass interval from $4 M_{\odot}$ to $1 M_{\odot}$ is $\alpha_{2}=2.7 \pm 0.5$, while in the mass range $\left(0.7<M<1 M_{\odot}\right)$ is $\alpha_{1}=1.8 \pm 0.2$ in marginal agreement with the Kroupa law. The LF of the observational data is compared with the best fit model in Fig. 8. When binaries are included, the age of the cluster becomes $\log ($ Age $)=8.3 \pm 0.2 \mathrm{yr}$. See the entries of Table 6 for the effect of binaries.

\subsubsection{NGC 290}

Inside a radius of $\sim 25^{\prime \prime}$ about 660 stars are found. Once more this radius is larger than the cluster core. Evolved stars brighter than $F 555 \mathrm{~W}=17.3 \mathrm{mag}$ are beyond the saturation level of the photometry. To cope with this we integrate our data for the red giants with those by OGLE II, whose photometry is converted in the $F 555 W$ and $F 814 W$ pass-bands, as described in the previous sections. Mounting the two sets of data has been made using equal areas in the two frames. No stars are missing in the HST photometry at the turnoff level $F 555 W=18$. This ensures that no spurious effects due to the use of ground-based and HST photometry influences the age determination. The top left panel of Fig. 4 presents the composite CMD.

As for the other two clusters, the preliminary inspection of the CMD provides estimates of age and metallicity which is then refined by the Monte Carlo technique. The automatic fit gives $\log ($ Age $)=7.8 \pm 0.5$ yr with $\chi^{2}=1.5$, metallicity $Z=0.003 \pm$ 0.002 , and $E(F 555 W-F 814 W)=0.15 \pm 0.05$. The IMF slope gives $\alpha_{1}=2.2 \pm 0.2$ in the mass range $0.7 M_{\odot} \leq M \leq 1 M_{\odot}$, and $\alpha_{2}=2.7 \pm 0.4$ in the mass range $M \geq 1 M_{\odot}$. Figure 4 presents the CMD of NGC 290 compared with our best fit isochrone. Figure 9 presents the observational LF compared with the best fit model. An older age is found when $30 \%$ of binary stars are included: $\log ($ Age $)=8.0 \pm 0.3 \mathrm{yr}$. Once again see the entries of Table 6 for the effect of binaries on the IMF parameters.

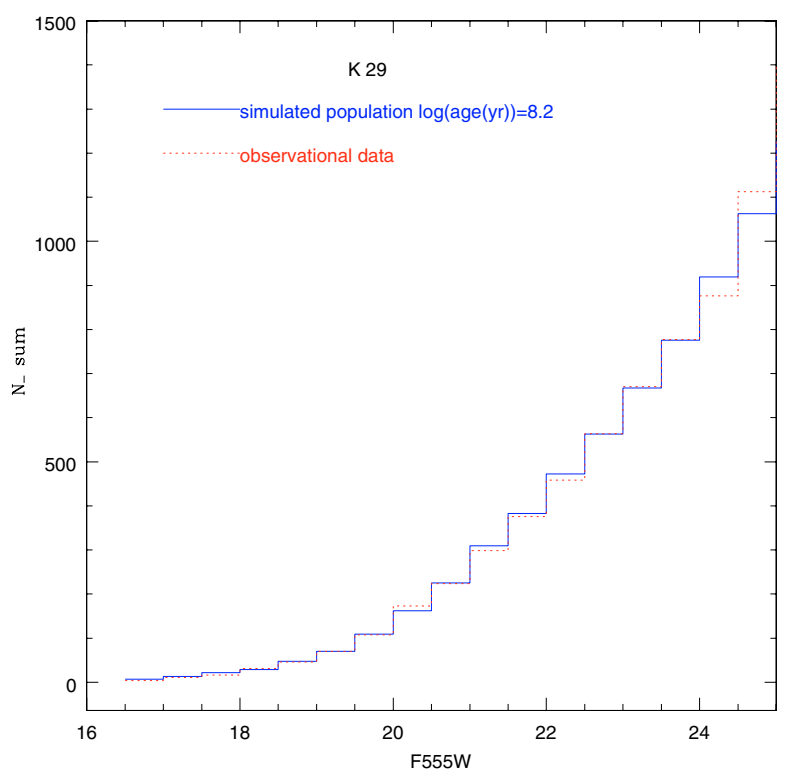

Fig. 8. LF of K 29 fitted with a population of $\log ($ Age $)=8.2 \mathrm{yr}$.

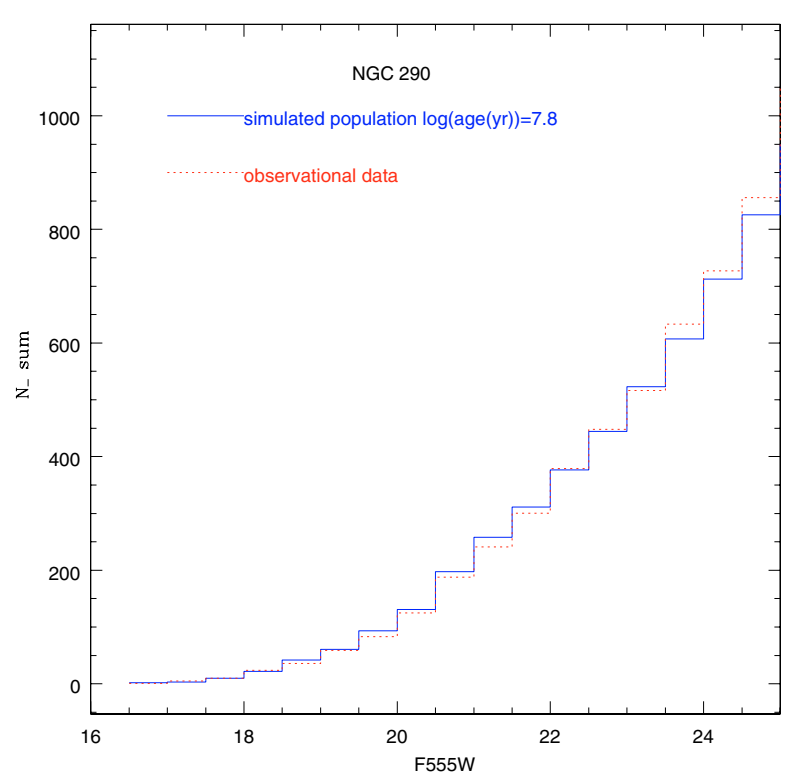

Fig. 9. LF of the cluster NGC 290 fitted with a simulated population having $\log ($ Age $)=7.8 \mathrm{yr}$.

\subsection{Revisiting an old question: convective overshoot}

Becker \& Mathews (1983), comparing observational and synthetic CMDs of NGC 1866, first noticed that classical stellar models they used predicted a number of red giants larger than observed, and a smaller number of main sequence stars in turn as compared to the observations. The same authors suggested that a more careful treatment of core convection (i.e. larger convective cores) could remove the discrepancy. A number of subsequent studies confirmed this idea and demonstrated that the inclusion of overshooting in the description of convective motions could reproduce the correct ratio of the number of evolved stars $N_{\mathrm{R}}$ over the number of main sequence stars, $N_{\mathrm{MS}}$. Chiosi et al. (1989a), in particular, clearly showed that the overshooting scheme, by reducing the ratio between the lifetime of the stars in the He-burning and the $\mathrm{H}$-burning phase, $t_{\mathrm{He}} / t_{\mathrm{H}}$, offers a good and simple solution to the problem. This conclusion was also 
reinforced by Lattanzio et al. (1991), who analysed the CMD of NGC 1866 by means of overshooting and classical models.

The classical theory of stellar structure predicts that in a star whenever the condition

$\nabla_{\mathrm{r}} \leq \nabla_{\mathrm{a}}$

is violated the region becomes unstable to convective motions and fully mixed. This region is surrounded by stable radiative layers. This is only an approximation: what happens physically at the border region passing from unstable to stable conditions? If we define the Schwarzschild core as the region at border of which the acceleration of convective bubbles vanishes there is still a shell above in which the velocity is not zero (the so-called overshoot region). The thickness, the thermal properties, and mixing efficiency in the overshooting region are still a matter of discussion. In any case, it is worth recalling that first convective overshoot is a consequence of the inertia principle, so that neglecting its existence would not be physically sound, second it is quite common in Nature (Deardorff et al. 1969). As demonstrated in a number of studies, including numerical simulations (Freytag et al. 1996), the penetration depth of convective elements into a formally stable region represents a non-negligible fraction of the size of the unstable zone. In addition to this, there is a number of astrophysical situations in which the hypothesis of substantial convective overshoot has been found to offer better and more elegant solutions than other explanations (see Bertelli et al. 1986a; Chiosi et al. 1992). Among others, suffice it to recall here the so-called mass discrepancy of Cepheid stars (Bertelli et al. 1986b; Chiosi et al. 1992). The first studies of this subject were by Shaviv \& Salpeter (1973); Prather \& Demarque (1974); Maeder (1975); Cloutman \& Whitaker (1980b); Bressan et al. (1981); Stothers \& Chin (1981), followed over the years by more sophisticated formulations standing on turbulence theories (Cloutman \& Whitaker 1980a; Xiong 1980) and fluid hydrodynamics (Canuto \& Mazzitelli 1991; Canuto et al. 1996; Unno \& Kondo 1989; Canuto 2000). The ballistic description long ago proposed by Bressan et al. (1981) is particularly suitable for the calculation of stellar models. It has been proved to best reproduce the numerical results of laboratory fluid-dynamics simulations (Zahn 1991). The Bressan et al. (1981) procedure will be shortly summarized below.

Despite all this, it has been often argued that unresolved binaries could mimic the effects of convective overshoot and easily account for the low ratio of evolved stars to main sequence stars observed in the young LMC clusters. This hypothesis has been investigated by many authors, both in open clusters of the Milky Way (see Carraro et al. 1994) and young clusters of the LMC (Chiosi et al. 1989a,b; Vallenari et al. 1992) with somewhat contrasting results. Worth of mention is the study by Testa et al. (1999) of NGC 1866, who introducing about $30 \%$ of binary and using the classical models of Dominguez et al. (1999) came to the conclusion that convective overshooting is not required. The subsequent analysis of Barmina et al. (2002) of the same cluster carried out on the Testa et al. (1999) data, discovered that the correction for completeness applied by Testa et al. (1999) was not correct thus invalidating their conclusions. In addition to this, they performed a systematic analysis of the effects of binary and stochastic fluctuations in the IMF, and came to the opposite conclusion: convective overshoot in unavoidable.

Here we have three clusters whose turn-off mass is about $4 M_{\odot}$ so it might be worth of interest to contribute further to this subject. In order to check which model better reproduces the data we need to have different sets of stellar populations. They are the two sets with convective overshoot, i.e. the models by Girardi et al. (2002) and those by Pietrinferni et al. (2004) with a different prescription so that comparison is possible.

As already recalled, the ballistic description of convective overshoot by (Bressan et al. 1981) is particularly suited to stellar models. The algorithm adopts a non-local treatment of convection in the context of the mixing-length theory (MLT) by Böhm-Vitense (1958): it looks for the layers where the velocity of convective elements (accelerated by the buoyancy force in the formally unstable regions) gets zero into the surrounding stable regions, then adopts a suitable temperature stratification in the overshoot regions, and finally assumes straight mixing over-there. Since the Bressan et al. (1981) formalism makes use of the MLT, it expresses the mean free path of the convective elements as $l=\Lambda_{\mathrm{c}} \times H_{\mathrm{p}}$ where $H_{\mathrm{p}}$ is the local pressure scale height. According to this definition adopted by Bressan et al. (1981) $\Lambda_{\mathrm{c}}$ represents the overshoot distance across the Schwarzschild radius in units of the pressure scale height. In Bressan et al. (1981) $\Lambda_{c}$ was assumed to be the same for all stellar masses. Over the years, this was slightly modified to treat overshoot in that particular range of mass which sees the transition from radiative core $\mathrm{H}$-burning to convective core H-burning and during this phase the convective core starts small, grows to a maximum and then decreases. In this mass interval $\Lambda_{\mathrm{c}}$ is not kept constant but let vary with mass according to the recipe in Girardi et al. (2000):

1) $\Lambda_{\mathrm{c}}=0$ for $M \leq 1.0 M_{\odot}$ (when the core is radiative);

2) $\Lambda_{\mathrm{c}}=M / M_{\odot}-1.0$ for $1 M_{\odot}<M \leq 1.5 M_{\odot}$;

3) $\Lambda_{\mathrm{c}}=0.5$ for $M>1.5 M_{\odot}$.

The last value stands also for the whole He-burning phase.

Pietrinferni et al. (2004) adopt a similar prescription:

1) $\Lambda_{\mathrm{c}}=0$ for $M \leq 1.1 M_{\odot}$ (when the core is radiative);

2) $\Lambda_{\mathrm{c}}=\left(M / M_{\odot}-0.9\right) / 4$ for $1 M_{\odot}<M \leq 1.5 M_{\odot}$;

3) $\Lambda_{\mathrm{c}}=0.2$ for $M \geq 1.7 M_{\odot}$.

where we have to keep in mind that $\Lambda_{c}$ is "measured" from the Schwarzschild border. Therefore it corresponds to about half of the Girardi et al. (2000) definition. Considered these premises, the two sets of tracks are practically coincident. This finding also ensures that using the classical models by Pietrinferni et al. (2004) is safe as the input physics adopted by the two groups is nearly the same.

The analysis is made comparing the integrated luminosity function of main sequence stars normalized to the number of evolved stars (thereinafter NILF) first introduced by Chiosi et al. (1989a), which has been proved to be the only way to effectively discriminate between the two different evolutionary schemes. It is worth reminding the reader that the NILF is by definition proportional to the lifetime ratio $t_{\mathrm{H}} / t_{\mathrm{He}}$ (Chiosi et al. 1989a).

The NILF requires that the number of evolved stars truly belonging to the cluster is known. The task is not trivial because it requires an accurate decontamination by field stars. The procedure is as follows. First of all, we apply to the CMD of the cluster and field the correction for completeness and area coverage. Second in the area of the cluster and field CMD we perform star counts. We statistically subtract from the cluster counts a number of evolved stars as large as that in the field. What remains are the expected cluster evolved stars. Finally, we consider only NGC 265 because the small number of giant stars in NGC 290 and K 29 would introduce too large statistical uncertainties as it is evident from Table 7 where the number of giant stars is given. Evolved stars are defined as the stars brighter 
Table 7. Number of evolved stars in the cluster and field CMDs.

\begin{tabular}{lccccc}
\hline \hline ID & Radius(") & Cluster & Field & Final & Uncertainty \\
\hline NGC 265 & 30 & 51 & 17 & 34 & \pm 5 \\
K 29 & 22 & 19 & 9 & 10 & \pm 3 \\
NGC 290 & 25 & 9 & 2 & 7 & \pm 3 \\
\hline
\end{tabular}

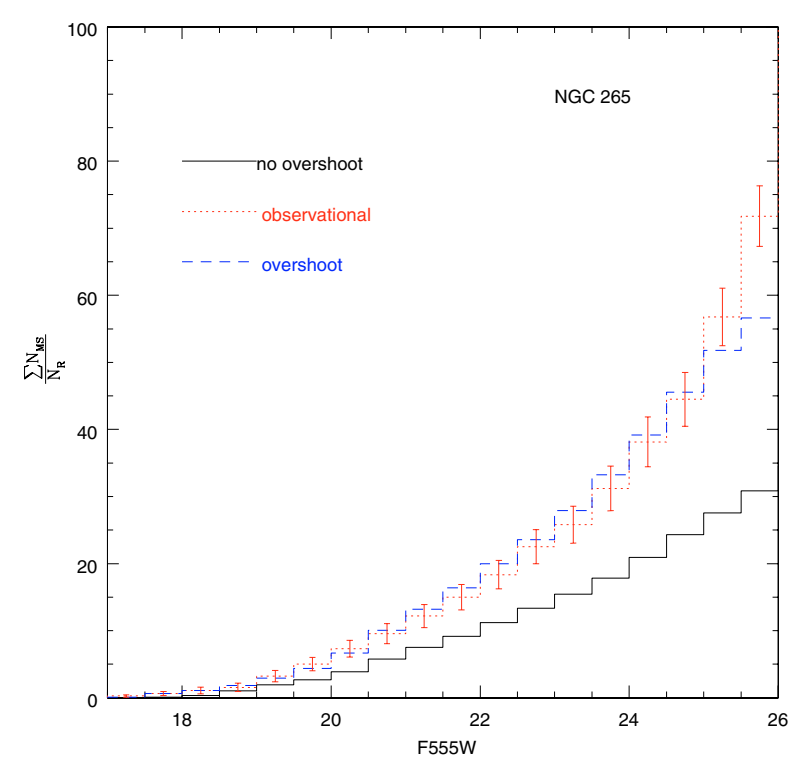

Fig. 10. Cluster NGC 265. We fit the NILF with canonical models taken from the BaSTI library and overshooting models from the Padova Library. Error bars are calculated taking into account photometric errors on the magnitudes (see text for details).

than $F 555 W=17$ and 16 for K 29 and NGC 290, respectively. In the case of NGC 265, evolved stars are the objects redder than $(F 555 W-F 814 W) \sim 0.4$ and brighter than $F 555 W=19$ mag.

We discuss the uncertainty due to the dependence on the adopted cluster area since mass segregation cannot be excluded inside the clusters. Recent estimate of the two-body relaxation time $t_{\mathrm{r}}$ inside MC clusters is given by Kerber \& Santiago (2006). They find values of $t_{\mathrm{r}}$ of the order of $10^{7}-10^{8} \mathrm{yr}$ inside the core radius, while for the whole clusters $t_{\mathrm{r}}$ is of a few Gyr. Although $t_{\mathrm{r}}$ does not exactly correspond to the time scale for stellar evaporation, this latter is expected to scale with it. The authors find significant variation of the present-day mass function exponent with the radius in the inner regions of the studied clusters as a result of the mass segregation in the core. If we assume that $t_{\mathrm{r}}$ inside the clusters studied in the present paper is of the same order and due to the fact that they have $\log ($ age $) \sim 8$, we come to the conclusion that they can suffer mass segregation in their core. Figure 11 shows the ratio of $\sum N_{\mathrm{MS}} / N_{\mathrm{R}}$ with the radius for the studied clusters for a limiting magnitude $F 555 W=25$. This ratio increases with the radius in the case of NGC 265, becoming constant at $30^{\prime \prime}$. This is due to the fact that red giants are more centrally concentrated than main sequence stars. In order to discuss the amount of convective overshoot in the NGC 265 stars, the region inside a radius of $30^{\prime \prime}$ should be considered, to minimize the effects of mass segregation.

The result for the NILF of NGC 265, shown in Fig. 10, seems to give an observational function that is intermediate between the two theoretical ones. This would mean that a certain amount of overshoot is needed to explain the data.

To cast light on the reliability of this result, we perform an analysis of the uncertainties affecting the NILF. We first

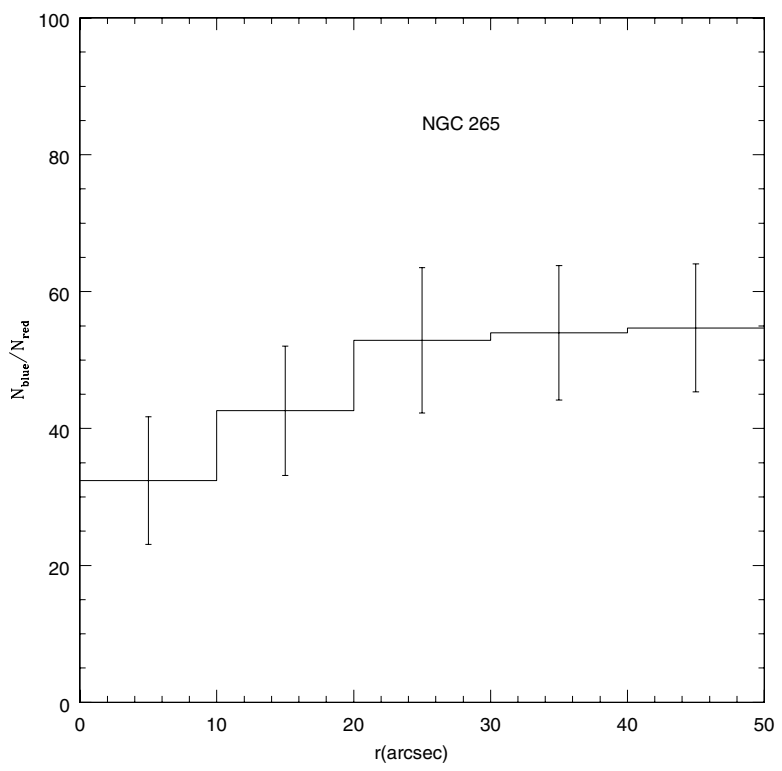

Fig. 11. The variation of the ratio $\sum N_{\mathrm{MS}} / N_{\mathrm{R}}$, briefly indicated as $N_{\text {blue }} / N_{\text {red }}$, with the radius for NGC 265 down to the limit magnitude $F 555 \mathrm{~W}=25$. The numbers take into account field subtraction and completeness correction. The error bars are calculated using the Poisson statistics.

discuss the effects of the uncertainties due to photometric errors, mainly acting on the integrated luminosity function $\sum N_{\mathrm{MS}}$. From crowding tests we draw the law that relates photometric errors and magnitudes. Then we compute how many stars fall in each bin of the histogram with a Monte Carlo simulation, giving to each star an equal probability to fall in the interval $v \pm \Delta v$ where $\Delta v$ is the relative photometric error. We repeat the test 100 times in order to build a sufficient statistical sample. Then we calculate for each of the bins the mean value and the root mean square that we consider as our uncertainty. The final error is calculated as the partial sum of single bin errors in the same way as we build the integrated luminosity function in Fig. 10.

Second, we evaluate the effect of the statistical uncertainties on the normalization factor $N_{\mathrm{RG}}$. As expected the steepness of the NILF is very sensitive to it. The experiment is made for NGC 265 data looking how the NILF varies at changing the number of red giants from 30 to 40 . The results are shown in Fig. 12. We notice first that the photometric errors do not significantly affect the NILF, second that the observations are in better agreement with overshoot models.

\section{History of star formation of the surrounding fields}

In this section we derive the star formation rate (SFR) of the field stars. Dynamical interaction of the SMC with nearby galaxies, i.e. the LMC and the Milky Way is a matter of discussion. Precise determinations of the age at which intense star formation activity took place would help to constrain the dynamical problem (Yoshizawa \& Noguchi 2003).

In Chiosi et al. (2006) we made use of the OGLE II data to derive the SFR in the SMC disk. We found evidence of several bursts of star formation younger than 2 Gyr together with episodes due to local phenomena. A burst is found at $200 \mathrm{Myr}$ which can be ascribed to the dynamical interaction between the Milky Way and the MCs. The limiting magnitude of the 


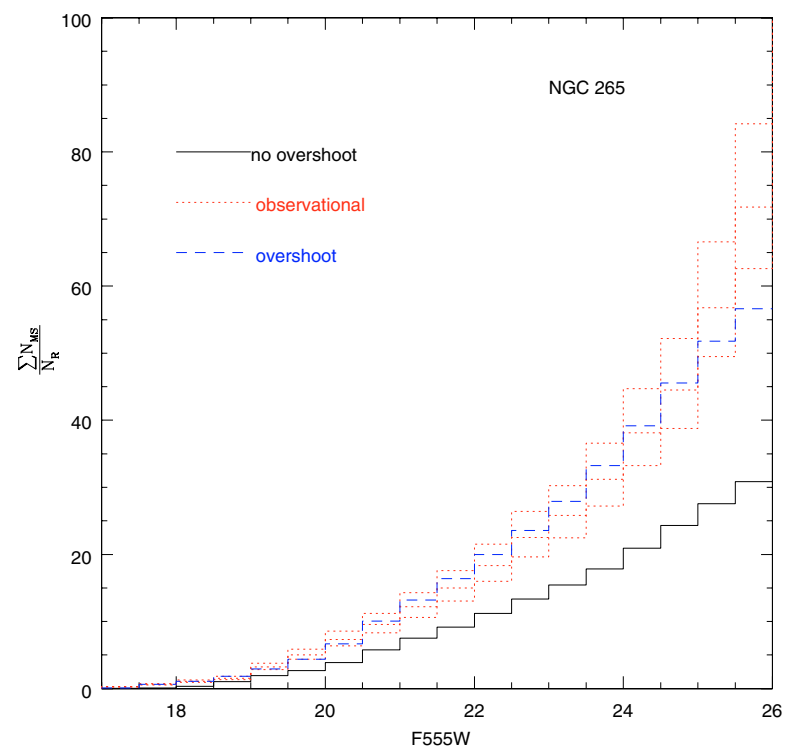

Fig. 12. The variation of NILF steepness at changing the number of red giants. The observational NILF (dotted line) is compared with theoretical counterpart from models with overshoot (dashed line) and classical models (solid line). Upper lines are normalized to 30 giants, intermediate to 34 , lower lines to 40 . We made these estimates on the consideration that the number of red giants is known within a certain uncertainty. After the subtraction of field red giants gives, for NGC 265 we get $34 \pm 5$ stars.

ground-based photometry did not allow any firm conclusion concerning older episodes. The deep limiting magnitude of the present HST-data allows the study of the star formation rate at old ages. In the following, first we present the method, then we discuss the star formation history of the field population in the studied regions.

To derive the field SFR, we make use of the whole area of the companion region of each cluster, and covers $202 \times 101$ arcsec. The large area of the regions allows a good statistics. Although in each case we are well outside the core radius of the associated cluster, some residual cluster contamination might still be present. This would result in a spurious peak at younger ages. However, as we are most interested in the determination of SFR at old ages, the spurious peak at very young ages would be less of a problem.

\subsection{The method}

The detailed description of the adopted method can be found in Chiosi et al. (2006). Here we recall a few points:

(1) To infer the SFR of a galaxy, theoretical CMDs in different age ranges are simulated. The simulations include the spread due to observational photometric errors, reddening, and the effect of photometric incompleteness. For each age bin, from 10000 to 15000 stars were generated down to the photometric completeness limit. The synthetic CMDs stand on the sets of stellar tracks, isochrones and single stellar populations by Girardi et al. (2002). We use $N=15$ stellar populations, whose ages and metal contents are listed in Table 8. Populations younger than $100 \mathrm{Myr}$ are under-sampled and will not be considered in the discussion. The entries of Table 8 also show that the component stellar populations are chosen in such a way that a suitable chemical enrichment history is assumed. The one adopted here is the chemical history found by Pagel \& Tautvaišienè (1999).
Table 8. Ages and metallicities of the synthetic populations in use.

\begin{tabular}{cc}
\hline \hline Age (Gyr) & $Z$ \\
\hline $0.08-0.12$ & 0.006 \\
$0.12-0.30$ & 0.006 \\
$0.30-0.40$ & 0.006 \\
$0.40-0.50$ & 0.006 \\
$0.50-0.60$ & 0.006 \\
$0.60-0.80$ & 0.006 \\
$0.80-1.00$ & 0.006 \\
$1.00-2.00$ & $0.001-0.006$ \\
$2.00-3.00$ & $0.001-0.003$ \\
$3.00-4.00$ & $0.001-0.003$ \\
$4.00-5.00$ & $0.001-0.003$ \\
$5.00-6.00$ & $0.001-0.003$ \\
$6.00-8.00$ & $0.001-0.001$ \\
$8.00-10.00$ & $0.001-0.001$ \\
$10.00-12.00$ & $0.001-0.001$ \\
\hline
\end{tabular}

(2) The relative contribution of different populations to the total CMD and luminosity/colour functions to be compared with the observational ones, in other words the past history of the SFR, is derived from a minimization algorithm that stands on the downhill simplex method (Harris \& Zaritsky 2004). It minimizes the $\chi^{2}$ function in a parameter-space having $N+1$ dimensions (the component stellar populations, here discretized to a finite number $N$ ). At each step of the simplex method, the local $\chi^{2}$ gradient is derived and a step in the direction of the gradient is taken, till a minimum is found. Care is paid to prevent settling of the simplex on local rather than global minima. Simplex is first started from a random position, then when a possible solution is obtained, it is re-started from a position very close to it. Finally, when a minimum is found, 30000 random directions are searched for a new minimum.

(3) To provide the required constraints to the minimization procedure, we split the observational CMD into a number of suitable magnitude-colour bins. Since the fit is mainly based on the main sequence, 28 bins of variable width are adopted. The bin width is $0.5 \mathrm{mag}$ from 14 to $19 \mathrm{mag}$, and $0.25 \mathrm{mag}$ from $19 \mathrm{mag}$ to $25 \mathrm{mag}$. For the red giant regions only three bins of suitable mag width are used, i.e. one for the sub-giant region, and one for the core He-burning clump, and one for the RGB/AGB stars. The adopted bins are shown in Fig. 13. The simulations are imposed to reproduce the relative percentages of stars in the various magnitude-colour bins.

\subsection{Results and discussion}

Star formation in the three fields shows several common features (see Figs. 15-17). In all the fields, the star formation was not continuous but proceeded in a number of bursts taking place at $0.3-0.4 \mathrm{Gyr}$, and between $3 \mathrm{Gyr}$ and $6 \mathrm{Gyr}$ ago.

The bursts at 3 and $0.4 \mathrm{Gyr}$ are temporally coincident with past peri-galactic passages of the SMC about the Milky Way. It is well known that the young field population presents an asymmetric structure biased toward the eastern LMC-facing side of the SMC. Crowl et al. (2001) find the same trend among the SMC populous clusters: those toward the eastern side tend to be younger and more metal rich than those on the western side. This is interpreted as the effect of the perturbations developed by the interaction of LMC-SMC-MW (see Chiosi et al. 2006, for a wider discussion). This is the kind of reshaping that a low mass disc galaxy ought to undergo after a few passages around a more massive galaxy (Pasetto et al. 2003). 


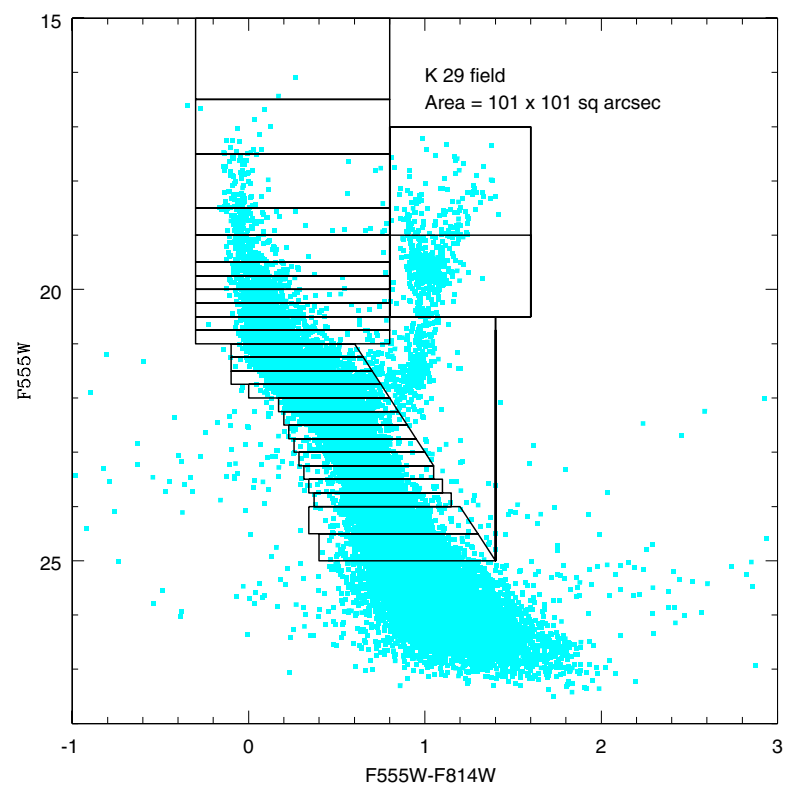

Fig. 13. The CM diagram of the field of K 29 with superimposed the grid adopted to derive the star formation. The same grid is used for the theoretical simulations. We count all the stars inside each box both in the synthetic and observational diagrams to construct the final SF history. The solution of the downhill simplex is the combination of coefficients (i.e. the mix of synthetic diagrams) that better fits the observational diagram.

While a larger consensus is reached in literature concerning the SFR at young ages and the influence of the interaction between the MCs and the Milky Way, still controversial results are obtained concerning the old population. In a pioneering work based on photographic plates, Gardiner \& Hatzidimitriou (1992) found that the bulk of the stellar population in the SMC is about 10 Gyr old. Harris \& Zaritsky (2004) find that $50 \%$ of the stars ever born in the SMC formed prior to $8.4 \mathrm{Gyr}$ ago. More precisely, the SMC formed relatively few stars between 8.4 and $3 \mathrm{Gyr}$ ago, but there was a rise in the mean SFR during the most recent 3 Gyr with bursts at ages of 2.5, 0.4, and 0.06 Gyr. Dolphin et al. (2001) find that the star formation has proceeded in a more continuous way, with a main episode between 5 and 8 Gyr. Noel et al. (2006) find a strong gradient with the galacto-centric distance and no significant star formation activity in the SMC at ages older than 6-9 Gyr.

The present result suggests a low efficiency of the star formation at old epochs. Using Monte Carlo simulations, we derive the uncertainty on this result. Small changes in the assumed lineof-sight SMC depth, extinction, age-metallicity relation result in an uncertainty of at least \pm 2 Gyr on the age of the components older than 3 Gyr. This is consistent with the magnitude bin size adopted to study the CMD. In fact a 0.25 mag uncertainty on the turnoff magnitude results in 2 Gyr age difference for a component of $5 \mathrm{Gyr}$, and $Z=0.004$. If we adopt a different metallicity, in the range 0.004 to 0.001 , the age-metallicity degeneracy is responsible of an additional uncertainty of about $0.5 \mathrm{Gyr}$.

To assess whether a significant component as old as or even older than $10 \mathrm{Gyr}$ is present in our fields, we compare the isochrones at changing metallicity with the CMD. The result is plotted in Fig. 14. The sub-giant branch width is not compatible with the presence of a significant component as old as or older than 10 Gyr.

This is in agreement with the age distribution of the clusters. In fact the SMC is known to have at least six populous clusters

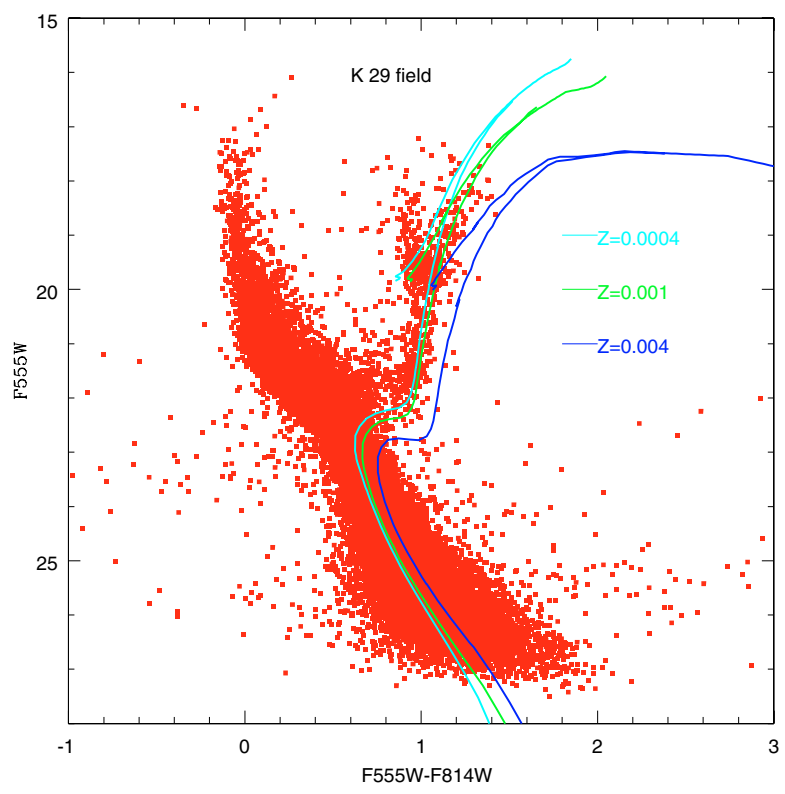

Fig. 14. The CM diagram of the field of $K 29$ with superimposed three isochrones of $10 \mathrm{Gyr}$ at different metallicity. The sub-giant branch width is not compatible with the presence of a significant population as old as or older than $10 \mathrm{Gyr}$ when the metallicity is ranging from $Z=0.0004$ to 0.004 .
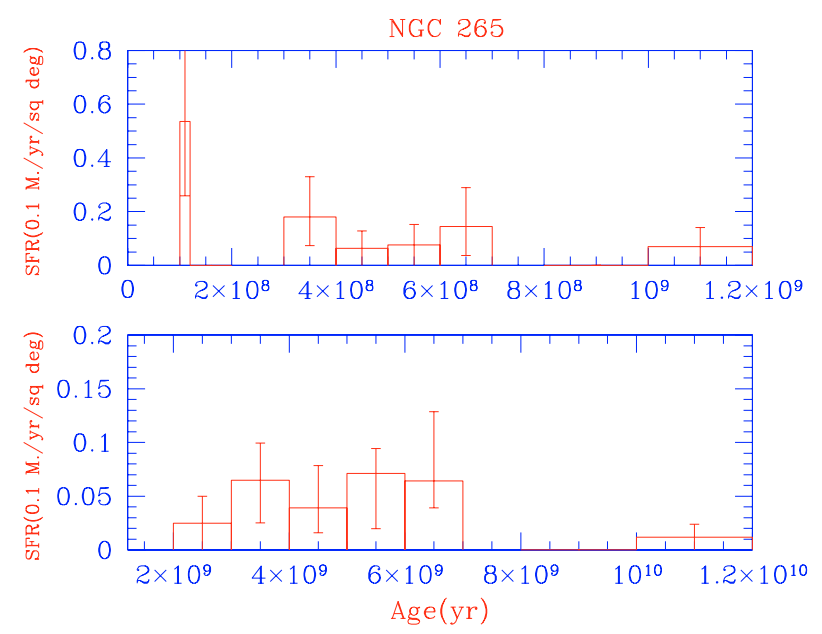

Fig. 15. Star formation history for the field of NGC 265. The age of the associated cluster corresponds to the burst of star formation at $300 \mathrm{Myr}$. The SFR is normalized to the area.

of intermediate age, namely in the range 5-9 Gyr, but only one true old object (NGC 121) having an age $>10$ Gyr is known in this galaxy (Stryker et al. 1985; Dolphin et al. 2001). The SFR of the field population in the LMC at older ages is not inconsistent with these results. In fact the majority of the authors find a strong population at intermediate ages (between 2-6 Gyr) (we quote among others Elson et al. 1997; Geha et al. 1998; Harris \& Zaritsky 1999, 2004; Olsen 1999; Dolphin 2000a; Javiel et al. 2005), but only in a few cases enhancements at older ages are found. Vallenari et al. (1996) find that in the regions East and South-East of the Bar the star formation had a sort of enhancement about 6-8 Gyr ago. Harris \& Zaritsky (2004) derive two main episodes of star formation at epochs younger than $2 \mathrm{Gyr}$ and older of 7 Gyr respectively, with a quiescent period in between. Smecker-Hane et al. (2002) find that the Disk SFR has been relatively smooth and continuous over the last $13 \mathrm{Gyr}$, 

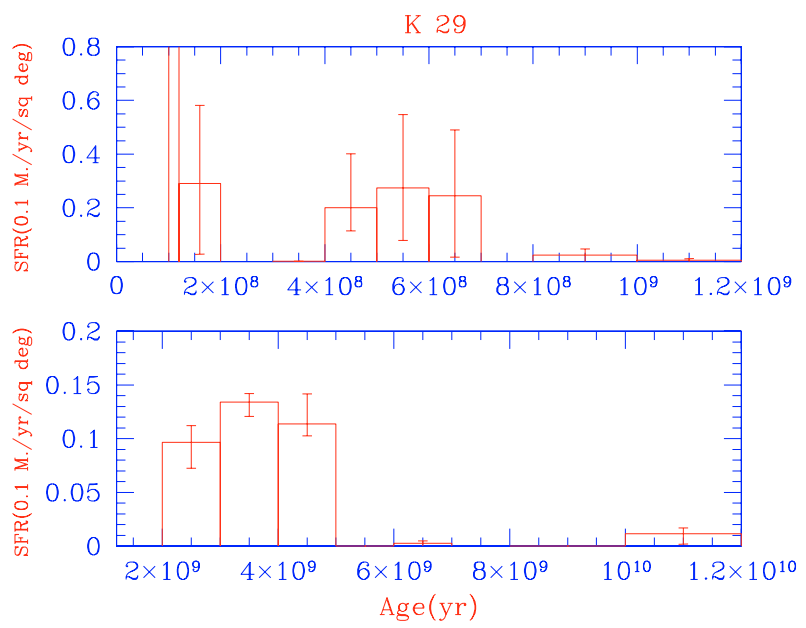

Fig. 16. Star formation history for the field of K 29. We can see bursts of star formation at ages 200-400 Myr, and between 3 Gyr and 5 Gyr. The age of the associated cluster corresponds to the first peak. The SFR is normalized to the area.
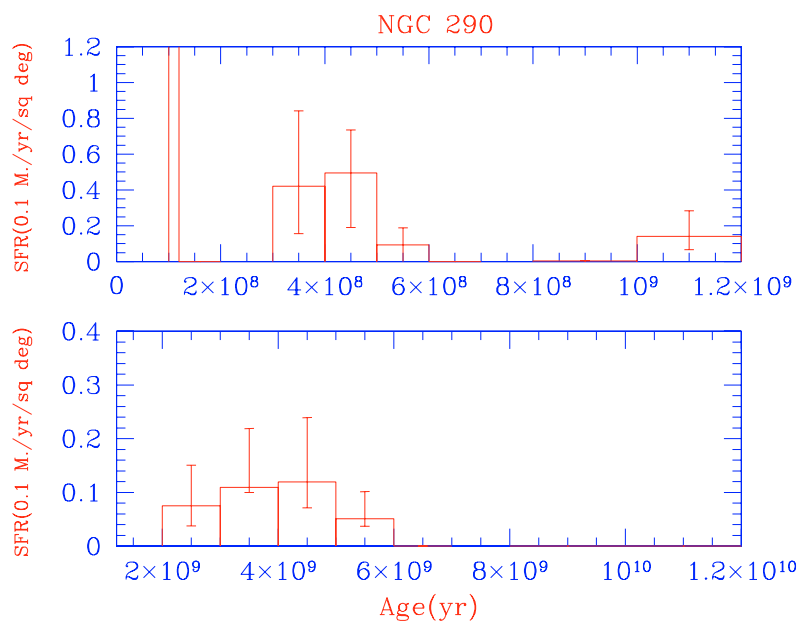

Fig. 17. Star formation history for the field of NGC 290. Main star formation episodes are at 300-400 Myr, and between 3 Gyr and 6 Gyr. Those episodes are common to the three areas suggesting a global triggering mechanism. The rate is normalized to the area.

while the Bar was dominated by SF episodes at intermediate ages, more precisely from 4 to 6 Gyr and 1 to 2 Gyr ago.

How can this be related to the interaction between the MCs and our Galaxy? Over the years our understanding of the problem has been much improving thanks to studies aimed at reproducing the properties of LMC and SMC by shaping their SFH by means of the mutual dynamical interactions in the triplet $\mathrm{LMC}+\mathrm{SMC}+\mathrm{MW}$. However, it is still not clear whether the MCs have existed in a binary state for most of their history (Fujimoto \& Murai 1984; Gardiner et al. 1994) or if their strong interaction happened just recently (Mathewson et al. 1986; Byrd et al. 1994; Bekki \& Chiba 2005; Yoshizawa \& Noguchi 2003). The solution is not unique owing to uncertainty in the initial conditions, and furthermore in many cases the MCs cannot keep their binary status for more than about 5-6 Gyr (Bekki \& Chiba 2005; Yoshizawa \& Noguchi 2003). Bekki et al. (2004) find that it is unlikely that the LMC and the SMC were a binary system more than 6 Gyr ago. Bekki \& Chiba (2005) pointed out that gravitational interactions do not necessarily influence in the same way the LMC and the SMC and that cluster and field star formed in different epochs. Since the LMC formed far from the Milky
Way (MW), no tidal trigger of the SF at old times is expected in this galaxy, while because of its smaller mass and its proximity, the SMC SFR was more influenced by MW tidal trigger. No cluster formation in the LMC took place as a consequence of the first perigalactic passage, $6.8 \mathrm{Gyr}$ ago, when instead the field star formation was enhanced. The present results concerning the SMC, suggest that at old ages, either there was no gravitational interaction between the two galaxies in agreement with Bekki et al. (2004), or the tidal interaction between the MCs, was not strong enough to trigger star formation episodes in the SMC.

\section{Summary and conclusions}

In this paper we have studied three clusters and companion fields of the SMC observed by E. Olszewski in year 2004 as part of the ACS/WFC program. The three clusters are located at the east side of HI super-shell 37A whose borders overlap a region of active star formation, most likely caused by the propagation of the gas pressure wave (Staveley-Smith et al. 1997; Stanimirovic et al. 1999). The ages assigned to the clusters on the basis of their CMD and luminosity function seem to be too old to be related to the dynamical age of the shell (Stanimirovic et al. 1999).

The ACS/WFC frame of each cluster is split in two parts, one containing the cluster itself and the other containing the companion field. Images are reduced with the usual DAOPHOT/ALLSTAR packages, completeness is calculated separately for the cluster and the field. We find the completeness limits ( $50 \%$ complete) for magnitudes $F 555 \mathrm{~W}=25$ for the clusters and $F 555 \mathrm{~W}=26$ for the fields.

In the ACS/WFC field containing the cluster we select a circular region inside which most of the cluster stars are located and derive the CMD and integrated luminosity function. The luminosity function and CMD are then compared to a library of synthetic luminosity functions and isochrones covering a large range of ages and metallicities. This yields a provisional value of the age and metal content best reproducing these two constraints. The results are then refined with the aid of population synthesis technique and the $\chi^{2}$-minimization algorithm. We find the following ages and metallicities: for the cluster NGC 265 the age is $\log ($ Age $)=8.5 \pm 0.3 \mathrm{yr}$ and the metallicity is $Z=0.004 \pm 0.003$ (corresponding to $[\mathrm{Fe} / \mathrm{H}] \sim-0.62$ ); for the cluster $\mathrm{K} 29$ the age is $\log ($ Age $)=8.2 \pm 0.2 \mathrm{yr}$ and the metallicity is $Z=0.003 \pm 0.002$ (corresponding to $[\mathrm{Fe} / \mathrm{H}] \sim-0.75$ ); for the cluster NGC 290 the age is $\log ($ Age $)=7.8 \pm 0.5 \mathrm{yr}$ and the metallicity is $Z=0.003 \pm 0.002$ (corresponding to $[\mathrm{Fe} / \mathrm{H}] \sim-0.75$ ).

We also estimate the slope of the IMF, especially at low masses, between $0.7 M_{\odot}$ and $1 M_{\odot}$. We check whether the Kroupa exponent, $\alpha_{1}=2.2$, is recovered in this range. We see that all the clusters have the minimum of $\chi^{2}$ function for $\alpha_{1}$ close but not identical to the Kroupa value. The values are $\alpha_{1}=2.4 \pm 0.4$ for NGC $265, \alpha_{1}=1.8 \pm 0.2$ for $\mathrm{K} 29$, $\alpha_{1}=2.2 \pm 0.2$ for NGC 290 . The differences are not significant at the $68 \%$ confidence level.

Limited to NGC 265, we also tested the theory of convective overshoot: we fitted the experimental NILF with two theoretical models, one including overshoot and the other without it taken from the BaSTI library. We can say that a certain amount of overshoot is needed in order to fit the experimental data.

Then we determine the past star formation history for the companion fields. The three determinations of the SF history for the three fields show several common features suggesting a common triggering mechanism over the scale length of interest here ( 700 pc). Main episodes are at 300-400 Myr, and between 3 Gyr and 6 Gyr. However the SFR was not very efficient at 
older ages. This result is in agreement with the cluster age distribution in the SMC and with the SFR at old ages in the LMC. This suggests that at old ages, the tidal interaction between the $\mathrm{MCs}$, if any, was not strong enough to trigger star formation episodes in both galaxies Finally, we point out that our determination of the past SF history is not precise for ages younger than about $100 \mathrm{Myr}$.

Acknowledgements. The authors thank C. Chiosi for reading the preliminary version of the manuscript, and G. Bertelli for many useful discussions and clarifications on the synthetic CMD generator. This study has been financially supported by the Department of Astronomy of the Padova University and the Padova Astronomical Observatory of the National Institute for Astrophysics (INAF).

\section{References}

Barmina, R., Girardi, L., \& Chiosi, C. 2002, A\&A, 385, 847

Becker, S., \& Mathews, J. 1983, AJ, 270, 155

Bedin, L. R., Cassisi, S., Castelli, F., et al. 2005, MNRAS, 357, 1038

Bekki, K., \& Chiba, M. 2005, MNRAS, 356, 680

Bekki, K., Couch, W. J., Beasley, M. A., et al. 2004, ApJ, 610, L93

Bertelli, G., Bressan, A., Chiosi, C., \& Angerer, K. 1986a, A\&AS, 66, 191

Bertelli, G., Bressan, A., Chiosi, C., Mateo, M., \& Wood, P. 1986b, ApJ, 412, 160

Böhm-Vitense, E. 1958, Z. Astroph., 46, 108

Bressan, A. G., Chiosi, C., \& Bertelli, G. 1981, A\&A, 102, 25

Byrd, G., Valtonen, M., McCall, M., \& Innanen, K. 1994, AJ, 107, 2055

Canuto, V. M. 2000, ApJ, 534, L113

Canuto, V. M., \& Mazzitelli, I. 1991, ApJ, 370, 295

Canuto, V. M., Goldman, I., \& Mazzitelli, I. 1996, ApJ, 473, 550

Caputo, F., Marconi, M., \& Ripepi, V. 1999, ApJ, 525, 784

Carraro, G., Chiosi, C., Bressan, A., \& Bertelli, G. 1994, A\&AS, 103, 375

Chiosi, C., Bertelli, G., \& Bressan, A. 1992, ARA\&A, 30, 235

Chiosi, C., Bertelli, G., Meylan, G., \& Ortolani, S. 1989a, A\&A, 219, 167

Chiosi, C., Bertelli, G., Meylan, G., \& Ortolani, S. 1989b, A\&AS, 78, 89

Chiosi, E., Vallenari, A., Held, E. V., Rizzi, L., \& Moretti, A. 2006, A\&A, 452, 179

Cloutman, L., \& Whitaker, R. W. 1980a, ApJ, 237, 900

Cloutman, L. D., \& Whitaker, R. W. 1980b, ApJ, 237, 900

Crowl, H. H., Sarajedini, A., Piatti, A. E., et al. 2001, AJ, 122, 220

Da Costa, G. S., \& Hatzidimitriou, D. 1998, AJ, 115, 1934

Deardorff, J., Willis, G., \& Lilly, D. 1969, Fluid Mech., 35, 7

Dolphin, A. E. 2000a, PASP, 112, 1397

Dolphin, A. E. 2000b, MNRAS, 313, 281

Dolphin, A. E., Walker, A. R., Hodge, P. W., et al. 2001, ApJ, 562, 303

Dominguez, I., Chieffi, A., Limongi, M., \& Straniero, O. 1999, ApJ, 524, 226

Elson, A., Sigurdsson, S., Davies, M., Hurley, J., \& Gilmore, G. 1998, MNRAS, 300,857

Elson, R. A. W. 1991, ApJS, 76, 185

Elson, R. A. W., Fall, S. M., \& Freeman, K. C. 1987, ApJ, 323, 54

Elson, R. A. W., Gilmore, G. F., \& Santiago, B. X. 1997, MNRAS, 289, 157

Freytag, B., Ludwig, H., \& Steffen, M. 1996, A\&A, 313, 497

Fujimoto, M., \& Murai, T. 1984, in Structure and Evolution of the Magellanic

Clouds, ed. S. van den Bergh, \& K. S. D. Boer, IAU Symp., 108, 115

Gardiner, L. T., \& Hatzidimitriou, D. 1992, MNRAS, 257, 195

Gardiner, L. T., Sawa, T., \& Fujimoto, M. 1994, MNRAS, 266, 567

Geha, M. C., Holtzman, J. A., Mould, J. R., et al. 1998, AJ, 115, 1045

Girardi, L., Bressan, A., Bertelli, G., \& Chiosi, C. 2000, A\&AS, 141, 371

Girardi, L., Bertelli, G., Bressan, A., et al. 2002, A\&A, 391, 195

Harris, J., \& Zaritsky, D. 1999, AJ, 117, 2831

Harris, J., \& Zaritsky, D. 2004, AJ, 127, 1531

Hill, A., \& Zaritsky, D. 2006, AJ, 131, 414

Hodge, P. 1986, PASP, 98, 1113
Hunter, D. A., Elmegreen, B. G., Dupuy, T. J., \& Mortonson, M. 2003, AJ, 126, 1836

Javiel, S. C., Santiago, B. X., \& Kerber, L. O. 2005, A\&A, 431, 73

Kerber, L. O., \& Santiago, B. X. 2006, A\&A, 452, 155

King, I. 1962, AJ, 67, 471

Kroupa, P. 2000, in Astronomische Gesellschaft Meeting Abstracts, ed. R. E. Schielicke, 11

Lattanzio, J., Vallenari, A., Bertelli, G., \& Chiosi, C. 1991, A\&A, 250, 340

Lisenfeld, U., \& Ferrara, A. 1998, ApJ, 496, 145

Mackey, A. D., \& Gilmore, G. F. 2003, MNRAS, 338, 120

Maeder, A. 1975, A\&A, 43, 61

Mathewson, D. S., Ford, V. L., \& Visvanathan, N. 1986, ApJ, 301, 664

McLaughlin, D. E., \& van der Marel, R. P. 2005, ApJS, 161, 304

Mermilliod, J., \& Mayor, M. 1989, A\&A, 219, 15

Noel, N., Gallart, C., Costa, E., \& Mendez, R. A. 2006, Rev. Mex. Astron. Astrofiz., 26, 76

Olsen, K. A. G. 1999, AJ, 117, 2244

Olszewski, E. W., Suntzeff, N. B., \& Mateo, M. 1996, ARA\&A, 34, 511

Pagel, B. E. J., \& Tautvaišienè, G. 1999, Ap\&SS, 265, 461

Pasetto, S., Chiosi, C., \& Carraro, G. 2003, A\&A, 405, 931

Piatti, A. E., Santos, J. F. C., Clariá, J. J., et al. 2001, MNRAS, 325, 792

Pietrinferni, A., Cassisi, S., Salaris, M., \& Castelli, F. 2004, ApJ, 612, 168

Pietrzynski, G., \& Udalski, A. 1999, Acta Astron., 49, 157

Prather, M. J., \& Demarque, P. 1974, ApJ, 193, 109

Rachford, B. L., Snow, T. P., Tumlinson, J., et al. 2002, ApJ, 577, 221

Rafelski, M., \& Zaritsky, D. 2005, AJ, 129, 2701

Rich, R. M., Shara, M., Fall, S. M., \& Zurek, D. 2000, AJ, 119, 197

Riess, A., \& Mack, J. 2004, ISR ACS, 2004

Salpeter, E. E. 1955, ApJ, 121, 161

Sandage, A., Bell, R. A., \& Tripicco, M. J. 1999, ApJ, 522, 250

Shaviv, G., \& Salpeter, E. E. 1973, ApJ, 184, 191

Sirianni, M., Jee, M. J., Benítez, N., et al. 2005, PASP, 117, 1049

Smecker-Hane, T. A., Cole, A. A., Gallagher, J. S., \& Stetson, P. B. 2002, ApJ, 566,239

Stanimirovic, S., Staveley-Smith, L., Dickey, J. M., Sault, R. J., \& Snowden, S. L. 1999, MNRAS, 302, 417

Stanimirović, S., Staveley-Smith, L., \& Jones, P. A. 2004, ApJ, 604, 176

Staveley-Smith, L., Sault, R. J., Hatzidimitriou, D., Kesteven, M. J., \& McConnell, D. 1997, MNRAS, 289, 225

Stetson, P. B. 1994, in The Restoration of HST Images and Spectra - II, ed. R. J. Hanisch, \& R. L. White, 308

Storm, J., Carney, B. W., Gieren, W. P., et al. 2004, A\&A, 415, 531

Stothers, R., \& Chin, C.-W. 1981, ApJ, 247, 1063

Stryker, L. L., Da Costa, G. S., \& Mould, J. R. 1985, ApJ, 298, 544

Testa, V., Ferraro, F., Chieffi, A., et al. 1999, AJ, 118, 2839

Udalski, A., Szymanski, M., Kubiak, M., et al. 1998, Acta Astron., 48, 147

Unno, W., \& Kondo, M. 1989, PASJ, 41, 197

Vallenari, A., Chiosi, C., Bertelli, G., Meylan, S., \& Ortolani, S. 1991, A\&AS, 87,517

Vallenari, A., Chiosi, C., Bertelli, G., Meylan, G., \& Ortolani, S. 1992, AJ, 104, 1100

Vallenari, A., Chiosi, C., Bertelli, G., \& Ortolani, S. 1996, A\&A, 309, 358

Welch, D. L., McLaren, R. A., Madore, B. F., \& McAlary, C. W. 1987, ApJ, 321, 162

Weldrake, D. T. F., Sackett, P. D., Bridges, T. J., \& Freeman, K. C. 2004, AJ, 128,736

Wilkinson, M. I., Hurley, J. R., Mackey, A. D., Gilmore, G. F., \& Tout, C. A. 2003, MNRAS, 343, 1025

Wilson, C. P. 1975, AJ, 80, 175

Wolfire, M. G., Hollenbach, D., McKee, C. F., Tielens, A. G. G. M., \& Bakes, E. L. O. 1995, ApJ, 443, 152

Xiong, D. R. 1980, ChA, 4, 234

Yoshizawa, A. M., \& Noguchi, M. 2003, MNRAS, 339, 1135

Zahn, J. 1991, A\&A, 252, 179

Zaritsky, D., Harris, J., Thompson, I. B., Grebel, E. K., \& Massey, P. 2002, AJ, 123,855 